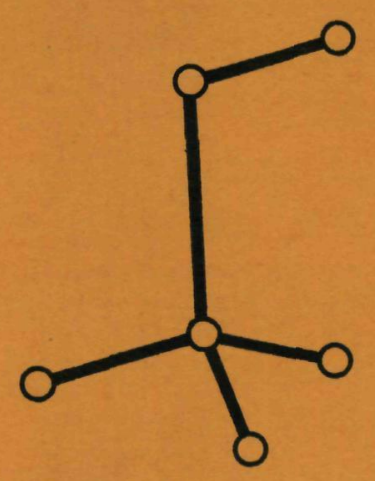

\title{
HYPERFINE STRUCTURE IN INTERNAL ROTOR MOLECULES
}

J. E. M. HEUVEL 

HYPERF INE STRUCTURE

IN INTERNAL ROTOR MOLECULES 
PROMOTOR: PROF.DR. A. DYMANUS 


\title{
HYPERFINE STRUCTURE IN INTERNAL ROTOR MOLECULES
}

\author{
PROEFSCHRIFT \\ TER VERKRIJGING VAN DE GRAAD VAN DOCTOR \\ IN DE WISKUNDE EN NATUURWETENSCHAPPEN \\ AAN DE KATHOLIEKE UNIVERSITEIT TE NIJMEGEN, \\ OP GEZAG VAN DE RECTOR MAGNIFICUS \\ PROF. DR. F. J. F. M. DUYNSTEE \\ VOLGENS BESLUIT VAN HET COLLEGE VAN DECANEN \\ IN HET OPENBAAR TE VERDEDIGEN \\ OP DONDERDAG 14 DECEMBER 1972 \\ DES NAMIDDAGS TE 2 UUR PRECIES \\ DOOR \\ JOHANM EDUARD MARIA HEUVEL.
}

GEBOREN TE LOBITH

1972

ENCELBARTS OFFSETDRUK - STOKKUM 
Gaarne wil ik allen bedanken die hebben bifgedragen tot het tot stand komen van dit proefschrift. Enkelen wil ik in het bifzonder vermelden.

Dr. J. Verhoeven, die mij heeft ingewijd in de experimentele techniek. De discussles die we voerden waren van grote waarde voor mij.

De Heren J. Holtkamp en E. van Leeuwen voor hun uitstekende technische hulp bij de uitvoering van het onderzoek.

Dr. J. Reuss voor de vele verhelderende discussies.

Degenen, die in hun afstudeerperiode stage gelopen hebben bif dit onderzoek. In het bijzonder Drs. A. Ellenbroek, die veel heeft geholpen bij de diverse metingen en de vaak moeizame berekeningen.

De leden van de werkgroep Atoom- en Molekuulfysika voor hun prettige samenwerking.

De dienst verlenende afdelingen in de personen van de Heren $J$. van Langen en $J$. van Bommel.

De figuren in dit proefschrift werden verzorgd door de afdeling illustratie onder leiding van de Heer J. Gerritsen.

Mijn vrouw wil ik graag bedanken voor $0 . a$. het typen van dit proefschrift. 
Aan mifn ouders

Aan Elly 
CHAPTER 1:

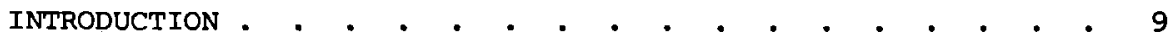

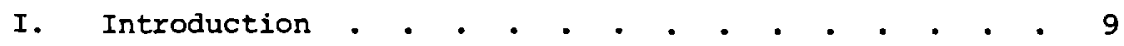

II. Hyperfine structure of rotational spectra . - . . 11

III. The experimental method . . . . . . . . . . 13

References • • • • • • • • • • • • • • • • 16

CHAPTER 2:

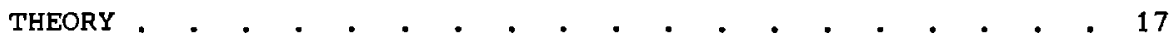

I. The Hamiltonian - . . . . . . . . . . . . 17

A. The Hamiltonian excluding hyperfine interactions . 18

B. The Hamiltonian including hyperfine interactions . 21

II. Methanol-11ke molecules . - . • . . - . . 28

III. The hyperfine structure - . . - . • . . . 36

A. The matrix elements . . . . . . . . . 36

B. The hyperfine coupling constants . . . . . 41

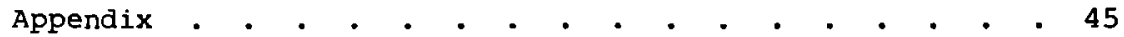

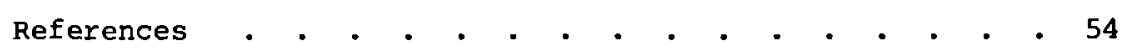

CHAPTER 3:

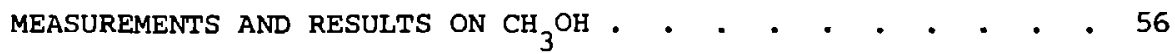

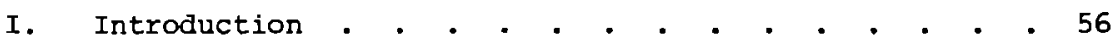

II. Spectrometers and experimental method . . . . . . 57

III. Experimental results and discussion . . . . . . 63 


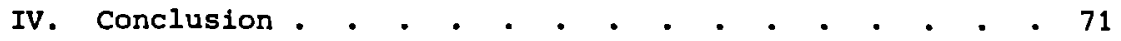

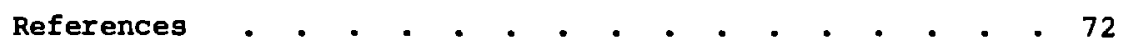

CHAPTER 4:

DISCUSSION $•$ •

I. Methanol . . . . . . . . . . . . . . . 73

II. Other molecules • • • • • • • • • • • • • 77

References • • • • • • • • • • • • • • • 78

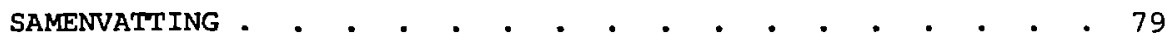



I. INTRODUCTION.

Hindered internal motion in molecules has been a subject of interest for long times. Molecules in which this type of motion occurs consist of two or more groups of nuclel which can move with respect to each other. The internal motion is generally not free, but hindered by potential barriers. The type and strength of these barriers determine the character of the internal motion. For high barriers the motion is a libration, because the probability to penetrate a barrier is small. The motion is an almost free rotation in the case of very low barriers. It is customary to call both types of internal motion "rotation", a custom whlch we shall observe in the following.

A considerable amount of information about the gross features of the internal rotation was and is being obtained from the rotational spectra in the microwave region. From the nature of the mlcrowave spectrum (strength and separation of librational satellite lines, splitting of the rotational lines of symmetric and asymmetric type of rotors) the helght and shape of the potential barriers can be determined in a semi-empirical way. The origin of the potentlal barrier is, however, not well understood. They are apparently caused by the interactions of two groups of nuclei and electrons.

It is well known that the hyperfine structure and the related 
molecular quantities are a very important source of information about the electronic structure and interactions in molecules. The present investigation was intended primarily to investigate the hyperfine structure of molecules with hindered internal rotation, both from theoretical as from experimental side. The goal is to examine how internal rotation influences the well known hyperfine interactions in rigid rotor molecules. The results might also be useful in the future for a better understanding of the phenomenon of internal rotation and in particular of the oxigin of the hindering potential barrier in terms of electronic structure.

All microwave spectra of internal rotors until the start of the present investigation were obtained at a rather low resolution and no information could be acquired about the hyperfine interactions in these molecules. Moreover, no theory was available that could be used as a basis for the interpretation of hyperfine spectra. Only recently Radford (1) reported the measurement of the $(\mathrm{J}, \mathrm{K}, \tau \pm)=(1,1,3+) \rightarrow(1,1,3-)$ transition of methanol $\left(\mathrm{CH}_{3} \mathrm{OH}\right)$ at $835 \mathrm{MHz}$ in a high resolution experiment. He did resolve the hyperfine structure, but made no attempt to analyse it.

The subject of the present investigations was also the methanol molecule. We have choosen this molecule because 1 t is one of the most intensively investigated internal rotors and, moreover, it is with the exception of hydrogen peroxide $\left(\mathrm{H}_{2} \mathrm{O}_{2}\right)$ also the simplest one. Many transitions of $\mathrm{CH}_{3} \mathrm{OH}$ have been reported in the centimeter and millimeter region, and most of them are well 1dentified. The barrier height and internal rotation wavefunctions have been calculated from these spectra. An additional importance of our 
Investigation stems from the recent discovery of methanol emission in the direction of the galactic center (2). This emission originates from transitions which were also subject of the present investigation. The hyperfine structure of these transitions is very important for the determination of the line shape and so called rest frequencies of the galactic emission lines.

II. HYPERFINE STRUCTURE OF ROTATIONAL SPECTRA.

In the absence of external electric and magnetic fields the main contribution of the hyperfine structure in rotational spectra originates in:

1) nuclear quadrupole interactions,

2) spin-rotation interactions, and

3) nuclear spin-spin interactions.

The first interaction arises from the coupling between the nuclear electric quadrupole moment with the gradient of the electric field at a given nucleus. The interaction is absent in ${ }^{12} \mathrm{CH}_{3} \mathrm{OH}$ since this molecule contains only nuclei with spin 0 or $\frac{1}{2}$. The deuterated species of methanol $\left(\mathrm{CH}_{3} \mathrm{OD}, \mathrm{CD}_{3} \mathrm{OH}, \mathrm{CD}_{3} \mathrm{OD}\right)$ were beyond the scope of the present investigation.

The nuclear spin-rotation interaction originates in the interactions of nuclear magnetic dipole moments with the effective magnetic field at the nucleus. This magnetic field is produced by the rotation of the nuclear frame and by the excitation of electrons into higher electronic states with non-zero angular momentum by the coupling between electronic motion and molecular rotation. The rotation has to be considered as composed of the overall-rotation of 
the molecule and the internal rotation. So there are two contributions to the spin-rotation interaction, namely the spin-overall rotation and the spin-internal rotation interaction. The strength of the spin-rotation interaction cannot be calculated in advance, but is expected to be of the order of $10 \mathrm{kHz}$, as it is in rigid rotor molecules.

The nuclear spin-spin interaction is the interaction between magnetic moments associated with nuclear spins. It consists theoretically of two parts: the direct- and the indirect, or electroncoupled spin-spin interaction. The latter interaction is very weak $(<1 \mathrm{kHz})$ and will be neglected in the following. The magnitude of the former interaction depends on the internuclear distances, which are not constant due to the internal rotation. The strength of this interaction can be calculated from the geometry of the molecule and the known internal rotation wavefunctions. For $\mathrm{CH}_{3} \mathrm{OH}$ it is of the order of $10 \mathrm{kHz}$.

At the start of the present investigation no theory of the hyperfine structure in internal rotor molecules was available. In Sect.2-I a Hamiltonian including hyperfine interactions is derived. This Hamiltonian is applicable not only to the methanol molecule, but also to other internal rotor molecules with only one internal degree of freedom. The last restriction is not essential and the hyperfine Hamlitonian is easily extended to molecules with more internal degrees of freedom. In the remaining part of Chap. 2 the structure of the hyperfine energy matrix and explicit expressions for the matrix elements and hyperfine coupling constants are introduced for methanol-like molecules. 
III. THE EXPERIMENTAL METHOD.

Rotational transitions of methanol cover a large frequency region, from a few hundreds of MegaHertz into the submillimeter region. The for the investigation most relevant transitions lie in the microwave region $(1-50 \mathrm{GHz})$.

The resolving power of a conventional microwave absorption spectrometer is generally insufficlent at frequencles higher than a few GigaHertz, because of the Doppler broadening of the lines. This broadening is considerably reduced in spectrometers using molecular beams. The ultimate resolution of such spectrometers is determined by the uncertainty principle of Helsenberg, if all broadening due to the Doppler effect and other instrumental effects is eliminated. The half-width (at half of the maximum intensity) of the line, $\Delta v$, due to the Helsenberg broadening in beam-spectrometers is given by:

$$
\Delta v \simeq \frac{\bar{v}}{2 L},
$$

where $\bar{v}$ is the most probable velocity of the molecules in the beam and $L$ is the length of the molecular path through the radiation field.

For the investigation of rotational transitions two types of beam-spectrometers enter into consideration

1) Beam-Absorption Spectrometer (BAS), and

2) Beam-Maser Spectrometer (BMS).

In the BAS the Doppler broadening is considerably reduced by sending a molecular beam perpendicular to the direction of propagation of the microwave radiation. In a practical realization of 
this principle a molecular beam is sent through the region between two plates of a parallel plate type absorption cell (Fig.3 chap.3). A diaphragm at liquid nitrogen temperature serves to trap the molecules not travelling in the proper direction. Information about the molecular energy levels is obtained from the measurement of the power absorbed by the molecules in the beam. The sensitivity of a BAS is proportional to the square of the frequency and becomes a serious problem below about $40 \mathrm{GHz}$. In this frequency region a BMS is an obvious choice for high resolution spectroscopy. In a BMS a considerable gain in sensitivity with respect to a BAS may be obtained by application of the maser principle Introduced by Gordon et $\underline{\text { al }}$ (3). Doppler broadening is eliminated by sending a molecular beam through a resonant structure (cavity or interferometer). However, a BMS equiped with a cylindrical microwave cavity cannot be used for frequencies above $30-40 \mathrm{GHz}$, since the dimensions of such cavities become too small for transmission of strong molecular beams. Although beam-maser spectrometers have been operated at frequencies as high as $200 \mathrm{GHz}$ using interferometers instead of conventional cavities, resolution is limited by the rather narrow width $(<10 \mathrm{~cm})$ of radiation distribution. With cylindrical cavities, lengths of $30 \mathrm{~cm}$ at $30 \mathrm{GHz}$ to $1 \mathrm{~m}$ at a few $\mathrm{GHz}$ can be used, yielding half-widths of $300-1000 \mathrm{~Hz}$.

The operation of the BMS is based on the phenomenon of stimulated emission of the molecules in the beam. The molecular beam passes through a state selector before entering the microwave cavity (Fig.1 Chap.3). The state selector consists usually of an electrostatic octupole which exerts a force on a molecule in the beam. 
The force depends on the Stark energy of the specific molecular state. All molecules in the lower of the two states involved in the transition are normally removed from the beam, while molecules in the upper state are deflected to the central axis of the state selector and enter the cavity. In the ideal situation, the molecules entering the cavity are all in the upper state from which they are stimulated to emit coherently by the electromagnetic field In the cavity.

The power $\Delta P$, emitted by the beam in the BMS or absorbed in the BAS, is proportional to:

$$
\Delta P \sim\left(n_{1}-n_{2}\right) \text { hv } P_{12}
$$

where $n_{1}$ and $n_{2}$ are the number of molecules in the upper and lower state, respectively, $P_{12}$ is the transition probabllity between the two states, and $v$ is the transition frequency. In the beam-maser spectrometer $n_{2}=0$ because of the state selection and hence:

$$
\Delta \mathrm{P} \sim \mathrm{n}_{1} \text { hV } \mathrm{P}_{12}
$$

If no state selection is employed, as in the BAS, the difference $n_{1}-n_{2}$ can be approximated by:

$$
n_{1}-n_{2}=-n_{1}\left(\frac{h v}{k T}\right)
$$

and hence:

$$
\Delta P \sim n_{1} \frac{h^{2} v^{2}}{k T} P_{12}
$$

The gain in sensitivity due to the state selection is therefore about (kT/hv), which is in the order of 300 for $\nu=20 \mathrm{GHz}$ and $T=300{ }^{0} \mathrm{~K}$. It depends, of course, on the stark effect of the in- 
volved molecular states.

The BMS is extremely small banded, and therefore the frequencies of the rotational transitions have to be known with an accuracy of about $200 \mathrm{kHz}$, before investigation with the BMS is possible. Therefore we first have determined accurately the frequencies of the rotational transitions we intended to investigate (4) These measurements were carried out with a conventional absorption spectrometer with stark modulation. At the same time the stark effect of the transition was determined, as it is important for the prospects of efficient state selection. In this way we discovered, for example, that the $(J, K, \tau \pm)=(5,1,3-) \rightarrow(5,1,3+)$ transition of methanol, which we intended to investigate, is completely unsultable for beam-maser spectroscopy because state selection is 1mpossible. The Stark effect of the rotational levels was also calculated and the agreement with the measurements was satisfying (ㅁ).

REFERENCES.

(1) H.E. Radford, private communication.

(2) J.A. Ball, C.A. Gottlleb, A.E. Lilley, and H.E. Radford, Astrophys.J. 162, L203 (1970).

(3) J.P. Gordon, H.J. Zeiger, and C.H. Townes, Phys.Rev. 99, 1264 (1955).

(4) J.E.M. Heuvel, Quarterly Report No 18 and 19, Atomic and Molecular Research Group, Katholieke Universiteit, N1jmegen (1968).

(5) A.W. Ellenbroek, Quarterly Report No 27, Atomic and Molecular Research Group, Katholieke Universiteit, Nijmegen (1970). 


\section{THEORY}

I. THE HAMILTONIAN.

The Hamiltonian of a ${ }^{1} \sum$ molecule with internal rotation can be written as: $\mathrm{H}=\mathrm{H}_{\mathrm{R}}+\mathrm{H}_{\mathrm{HF}}$, where $\mathrm{H}_{\mathrm{R}}$ is the Hamiltonian of the overall rotation of the molecule and $H_{H F}$ represents contributions to the energy because of hyperfine interactions originating in the spins of molecular nuclei (spin-spin, spin-rotation, spin-internal rotation). We restrict ourselves in this section to internal rotor molecules with only one internal degree of freedom $\left(\mathrm{CH}_{3} \mathrm{OH}\right.$ and $\mathrm{H}_{2} \mathrm{O}_{2}$ for example). If we choose a molecule-flxed system of reference *) $(x, y, z$, not necessarily the princlpal-axis system) with the origin in the center of mass, the overall velocity of a nucleus $K$ is given by:

$$
\dot{\bar{r}}_{K}=\left(\bar{\omega} \times \bar{r}_{K}\right)+\bar{v}_{K}
$$

Herein $\bar{r}_{K}$ is the radius vector of the $K$-th nucleus, $\bar{v}_{K}$ is the velocity of this nucleus relative to the molecule-fixed system, and $\bar{\omega}$ is the overall angular velocity of the molecule-fixed system relative to the space-fixed system. In internal rotor molecules with one internal degree of freedom, the velocity $\bar{v}_{K}$ of all nuclei is perpendicular to a certain axis $\bar{\lambda}$ through the $C M$ and proportional to $\frac{\partial a}{\partial t}$, $\alpha$ being the angle of internal rotation. In this case, for each nucleus $K$ a vector $\bar{\sigma}_{K}$ can be found such that:

*) We suppose that such a system can always be defined. 


$$
\overline{\mathrm{v}}_{\mathrm{K}}=-\dot{\alpha}\left(\bar{\lambda} \times \bar{\sigma}_{\mathrm{K}}\right)=-\dot{\bar{\alpha}} \times \bar{\sigma}_{\mathrm{K}}
$$

where $\dot{\bar{\alpha}}=\dot{\alpha} \bar{\lambda}$. The sign in the above expression is arbitrary, and depends on the definition of $\alpha, \bar{\lambda}$ and $\bar{\sigma}_{K}$. Equation (1) can be rewritten as:

$$
\dot{\bar{r}}_{\mathrm{K}}=\left(\bar{\omega} \times \overline{\mathbf{r}}_{\mathbf{K}}\right)-\left(\dot{\bar{\alpha}} \times \bar{\sigma}_{\mathbf{K}}\right)
$$

A. THE HAMILTONIAN EXCLUDING HYPERFINE INTERACTIONS.

The kinetic energy is given by:

$$
\begin{aligned}
\mathrm{T} & =\sum_{\mathrm{K}} \frac{1}{\frac{1}{2}} \mathrm{~m}_{\mathrm{K}}\left|\overline{\mathrm{r}}_{\mathrm{K}}\right|^{2}=\sum_{\mathrm{K}} \frac{1}{2} \mathrm{~m}_{\mathrm{K}}\left|\left(\bar{\omega} \times \overline{\mathrm{r}}_{\mathrm{K}}\right)-\left(\dot{\bar{\alpha}} \times \bar{\sigma}_{\mathrm{K}}\right)\right|^{2} \\
& =\frac{1}{2} \bar{\omega} \cdot \overline{\overline{\mathrm{I}}}{ }^{\mathrm{r}} \cdot \bar{\omega}-\bar{\omega} \cdot \overline{\bar{I}} \sigma \mathrm{r} \cdot \dot{\bar{\alpha}}+\frac{1}{2} \dot{\bar{\alpha}} \cdot \overline{\bar{I}} \sigma \sigma \cdot \dot{\bar{\alpha}},
\end{aligned}
$$

where the components $I_{g g}^{a b}$, of the inertia tensors $\overline{\bar{I}}^{r r}, \overline{\bar{I}}^{\sigma r}, \overline{\bar{I}}^{\sigma \sigma} \mathrm{de}-$ fined with respect to the molecular axes system $\left(g, g^{\prime}\right)$, are defined by :

$$
I_{g g^{\prime}}^{a b}=\sum_{K} m_{K}\left[\left(\bar{a}_{K} \cdot \bar{b}_{K}\right) \delta\left(g, g^{\prime}\right)-\left(\bar{a}_{K}\right)_{g}\left(\bar{b}_{K}\right)_{g^{\prime}}\right] .
$$

$\overline{\bar{I}}^{\mathrm{rr}}$ is the inertia tensor of the whole molecule and $\overline{\bar{I}}^{\sigma \sigma}$ is sometimes the inertia tensor of one part of the molecule $\left(\mathrm{CH}_{3} \mathrm{OH}\right)$. The Cartesian components of the total angular momentum $\bar{R}$ and the angular momentum $P$ conjugate to the angle $\alpha$ of internal rotation are:

$$
\begin{array}{lll}
R_{g} \equiv \frac{\partial T}{\partial \omega_{g}} & \text { or } & \bar{R}=\overline{\bar{I}}^{r r} \cdot \bar{\omega}-\dot{\alpha} \bar{I}^{\sigma r} \cdot \bar{\lambda}, \\
P \equiv \frac{\partial T}{\partial \dot{\alpha}} & \text { or } & P=-\bar{\omega} \cdot \overline{\bar{I}}^{\sigma r} \cdot \bar{\lambda}+\dot{\alpha} \bar{\lambda} \cdot \overline{\bar{I}}^{\sigma \sigma} \cdot \bar{\lambda} .
\end{array}
$$

By expressing the angular velocities $\dot{\alpha}$ and $\bar{\omega}$ in terms of the angu- 
lar momenta we get:

$$
\begin{aligned}
& \dot{\alpha}=2 F(\Pi+P), \\
& \bar{\omega}=2 \overline{\bar{A}} \bar{R}+2 F(\Pi+P) \bar{\rho},
\end{aligned}
$$

where :

$$
\begin{aligned}
& \overline{\bar{A}}=\frac{1}{2}\left(\overline{\bar{I}}^{\mathrm{rr}}\right)^{-1}, \\
& \bar{\rho}=2 \overline{\bar{A}} \cdot \overline{\bar{I}} \sigma r \cdot \bar{\lambda}, \\
& \Pi=(\bar{p} \cdot \bar{R}) \text {, } \\
& F=\frac{1}{2}\left[\bar{\lambda} \cdot \overline{\bar{I}}^{\sigma \sigma} \cdot \bar{\lambda}-\bar{\rho} \cdot \overline{\bar{I}} \mathbf{r r} \cdot \bar{\rho}\right]^{-1} \text {. }
\end{aligned}
$$

From Eqs. (5), (6) and Eq. (3) follows:

$$
T=\bar{R} \cdot \overline{\bar{A}} \cdot \bar{R}+F(\Pi+P)^{2}
$$

The Internal rotation is in general not free, but hindered by the interactions between the atoms of the molecule. We suppose that these Interactions can be described by a potential $V(\alpha)$, depending on the internal angle $a$. The Hamiltonian $H_{R}$ of the overall- and internal rotation is then:

$$
H_{R}=\bar{R} \cdot \overline{\bar{A}} \cdot \bar{R}+F(\Pi+P)^{2}+V(\alpha) .
$$

The angular momenta $\bar{R}$ and $P$ are associated with rotation of the nucle1 alone. The total angular momentum $\overline{\mathbf{J}}$ of the molecule, excluding nuclear spins is:

$$
\bar{J}=\bar{R}+\bar{L},
$$

where $\bar{L}$ is the angular momentum of the electrons. The latter momentum is zero in first order for ${ }^{1} \sum$ molecules, but not in second 
order. Similarly:

$$
\mathrm{p}=\mathrm{P}+\mathrm{L}_{\alpha},
$$

where $\mathrm{p}$ is the total angular momentum assoclated with internal rotation, and $L_{\alpha}$ the electronic contribution to $1 t$.

The rotational Hamiltonian in terms of the total and electronic angular momenta can be written in the form:

$$
\mathrm{H}_{\mathrm{R}}=(\overline{\mathrm{J}}-\overline{\mathrm{L}}) \cdot \overline{\bar{A}} \cdot(\overline{\mathrm{J}}-\overline{\mathrm{L}})+\mathrm{F}\left(\Gamma-\Lambda+\mathrm{p}-\mathrm{L}_{\alpha}\right)^{2}+\mathrm{V}(\alpha),
$$

with:

$$
\Gamma=(\bar{\rho} \cdot \bar{J}),
$$

and:

$$
\Lambda=(\bar{\rho} \cdot \bar{L}) \text {. }
$$

The Hamiltonian (Eq. (9)) can be written as a sum:

$$
\mathrm{H}_{\mathbf{R}}=\mathrm{H}_{0}+\mathrm{H}_{1} \text {, }
$$

where:

$$
H_{0}=\bar{J} \cdot \overline{\bar{A}} \cdot \bar{J}+F(\Gamma+p)^{2}+V(\alpha)
$$

is the zeroth order contribution, which is independent of the electronic angular momenta, and

$$
\mathrm{H}_{1}=-2 \overline{\mathrm{L}} \cdot \overline{\bar{A}} \cdot \overline{\mathrm{J}}+(\Gamma+p) \xi
$$

with :

$$
\xi=-2 F\left(\Lambda+L_{\alpha}\right) \text {, }
$$

is the first order contribution, containing only terms linear in the electronic orbital angular momenta $\overline{\mathrm{L}}$ and $\mathrm{L}_{\alpha}$. The terms quadratic in $\bar{L}$ and $L_{\alpha}$ can be neglected, because they are independent of the rotational quantum numbers. 
B. THE HAMILTONIAN INCLUDING HYPERFINE INTERACTIONS.

In the absence of external magnetic fields the magnetic hyperfine structure originates in interactions (1) between the magnetic moments of the nuclei, and (2) between the nuclear magnetic moments and the magnetic field produced at their positions by the motion of molecular electrons and nuclel. The first interaction is the familiar spin-spin interaction described by a Hamiltonian $H_{S S}$. The electronic (e) and nuclear (n) contribution to the second interaction, the spin-rotation interaction, is described by the Hamiltonian $\mathrm{H}_{\mathrm{SR}}^{(\mathrm{e})}$ and $H_{S R}^{(n)}$, respectively. The total hyperfine Hamiltontan 1s:

$$
H_{H F}=H_{S R}^{(e)}+H_{S R}^{(n)}+H_{S S} \text {. }
$$

The "classical" expressions (in MKS-units) for the individual Hamiltonians are $(\underline{1}, \underline{2}, \underline{3}, \underline{4}):$

$\mathrm{H}_{\mathrm{SR}}^{(e)}=\frac{e \mu_{0} \mu_{N}}{4 \pi} \sum_{1} \sum_{K} g_{K} r_{1 K}^{-3}\left[\bar{r}_{i K} \times\left(\bar{v}_{1}-\gamma_{K} \bar{v}_{K}\right)\right] \cdot \bar{I}_{K}$,

${ }_{\mathrm{SR}}^{(\mathrm{n})}=\frac{-\mathrm{e} \mu_{\mathrm{O}} \mu_{\mathrm{N}}}{4 \pi} \sum_{\mathrm{K}} \sum_{L \neq K} \mathrm{z}_{\mathrm{L}} \mathrm{g}_{\mathrm{K}} \mathrm{r}_{\mathrm{LK}}^{-3}\left[\overline{\bar{r}}_{\mathrm{LK}} \times\left(\overline{\mathrm{v}}_{\mathrm{L}}-\gamma_{\mathrm{K}} \overline{\mathrm{v}}_{\mathrm{K}}\right)\right] \cdot \overline{\mathrm{I}}_{\mathrm{K}}$,

$H_{S S}=\frac{\mu_{0} \mu_{N}^{2}}{4 \pi} \sum_{K} \sum_{L<K} g_{L} g_{K} r_{L K}^{-3}\left[\left(\bar{I}_{L} \cdot \bar{I}_{K}\right)-3 r_{L K}^{-2}\left(\bar{I}_{K} \cdot \bar{r}_{L K}\right)\left(\bar{I}_{L} \cdot \bar{r}_{L K}\right)\right]$

In these expressions $\bar{r}_{\mathbf{s}}$ and $\bar{v}_{\mathbf{s}}$ denotes the radius- and velocity vector, respectively, of particle $s$ ( 1 for electrons, $K$ and $L$ for nuclei) relative to the molecular center of mass, which is assumed to be the center of mass (CM) of all nuclei, $\bar{r}_{i K}=\bar{r}_{1}-\bar{r}_{K}$ and $\bar{r}_{L K}=\bar{r}_{L}-\bar{r}_{K}, r_{I K}=\left|\bar{r}_{I K}\right|$ and $r_{L K}=\left|\bar{r}_{L K}\right|$. The nucleus $K$ is supposed to have mass $M_{K}$, charge $z_{K} e$ (e positive), spin $I_{K}$, and 
nuclear magnetic moment $g_{K^{\mu}} \bar{I}_{K}$, with $g_{K}$ the nuclear g-factor and $\mu_{N}$ the nuclear magneton. The Thomass precession factor is given by:

$$
\gamma_{K}=-\frac{z_{K}^{M} p}{g_{K}^{M}}
$$

with $M_{p}$ the proton mass.

The Hamiltonians $\mathrm{H}_{\mathrm{SS}}, \mathrm{H}_{\mathrm{SR}}^{(\mathrm{e})}$ and $\mathrm{H}_{\mathrm{SR}}^{(n)}$ can be expressed as products of tensors of rank 1 and 2 .

\section{(i) Spin-spin interaction.}

This case is formally identical to that of a rigid rotator without internal rotation. The reason is that interaction between nuclear splns is independent of velocities of the nuclei and depends only on their positions. So we have:

with:

$$
\mathrm{H}_{\mathrm{SS}}=\sum_{\mathrm{K}} \sum_{\mathrm{L}<\mathrm{K}} \overline{\mathrm{I}}_{\mathrm{K}} \cdot \overline{\overline{\mathrm{D}}}_{\mathrm{KL}} \cdot \overline{\mathrm{I}}_{\mathrm{L}},
$$

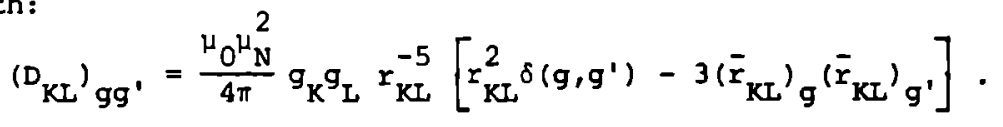

The angle $\alpha$ will enter into the calculation through the relative positions of the nuclei.

(ii) Spin-rotation interaction.

By substituting the expression for $\bar{v}_{\mathrm{K}}$ given in Eq. (1) into Eq. (12b) we obtain:

$H_{S R}^{(n)}=\frac{-\mu_{0}^{\mu} N}{4 \pi} \sum_{K L F K} \sum_{L} z_{L} g_{K} x_{L K}^{-3}\left\{\bar{r}_{L K} \times\left[\left(\bar{\omega}_{K} \times \bar{z}_{L K}\right)-\left(\dot{\bar{\alpha}} \times \bar{\Sigma}_{L K}\right)\right]\right\} \cdot \bar{I}_{K}$,

with: 


$$
\begin{aligned}
& \bar{z}_{L K}=\bar{r}_{L}-\gamma_{K} \bar{r}_{K}, \\
& \bar{\Sigma}_{L K}=\bar{\sigma}_{L}-\gamma_{K} \bar{\sigma}_{K} .
\end{aligned}
$$

Since we are dealing with ${ }^{1} \Sigma$ molecules, we approximate (in first order) the expressions for $\bar{\omega}$ and $\dot{\alpha}$ given in Eqs. (5) and (6) by:

$$
\begin{aligned}
& \bar{\omega}=2 \overline{\bar{A}} \bar{J}+2 F(\Gamma+p) \bar{p}, \\
& \bar{\alpha}=2 F(\Gamma+p) \bar{\lambda} .
\end{aligned}
$$

With this approximation Eq. (14) reduces to:

$$
\begin{aligned}
H_{S R}^{(n)}=\frac{-{ }_{0} \mu_{N}}{2 \pi} \sum_{K} \sum_{L \neq K} z_{L} g_{K} r_{L K}^{-3}\left\{\bar{r}_{L K} \times\left[(\overline{\bar{A}} \bar{J}) \times \bar{z}_{L K}\right]+\right. \\
\left.+(\Gamma+p) \bar{d}_{L K}^{n}\right\} \cdot \bar{I}_{K} .
\end{aligned}
$$

with:

$$
\overline{\mathrm{d}}_{L K}^{\mathrm{n}}=\mathrm{F}\left\{\overline{\mathbf{r}}_{L K} \times\left[\left(\bar{\rho} \times \bar{z}_{L K}\right)-\left(\bar{\lambda} \times \bar{\varepsilon}_{L K}\right)\right]\right\},
$$

or:

$$
H_{S R}^{(n)}=\sum_{K}\left\{\bar{I}_{K} \cdot \overline{\bar{N}}_{K}^{n} \cdot \bar{J}+(\Gamma+p)\left(\bar{I}_{K} \cdot \bar{d}_{K}^{n}\right)\right\},
$$

with:

$$
\begin{aligned}
& \left(N_{K^{\prime}}^{n}\right)^{\prime} g^{\prime}=\frac{-\mu_{0} \mu_{N}}{2 \pi} g_{K} \sum_{L \neq K} z_{L} r_{K L}^{-3} \sum_{g^{\prime \prime}} A_{g^{\prime} g^{\prime \prime}}\left\{\left(\bar{r}_{L K} \cdot \bar{z}_{L K}\right) \delta\left(g, g^{\prime \prime}\right)-\right. \\
& \left.-\left(\bar{z}_{L K}\right)_{g}\left(\bar{r}_{L K}\right)_{g "}\right), \\
& \bar{d}_{K}^{n}=\frac{-e{ }_{O}^{\mu} N}{2 \pi} g_{K} \sum_{L \neq K} r_{K L}^{-3} \bar{d}_{L K}^{n} .
\end{aligned}
$$

By combining Eqs.(11),(12),(13) and (16) we untain:

$$
\mathrm{H}=\mathrm{h}_{0}+\mathrm{h}_{1}+\mathrm{h}_{2} \text {, }
$$

with: 


$$
h_{0}=H_{0}=\bar{J} \cdot \overline{\bar{A}} \cdot \bar{J}+F(\Gamma+p)^{2}+V(a),
$$

$$
\begin{aligned}
\mathrm{h}_{1} & =\mathrm{H}_{\mathrm{SR}}^{(\mathrm{n})}+\mathrm{H}_{\mathrm{SS}}= \\
& =\sum_{K}\left\{\overline{\mathrm{I}}_{\mathrm{K}} \cdot \overline{\overline{\mathrm{N}}}_{\mathrm{K}}^{\mathrm{n}} \cdot \overline{\mathrm{J}}+(\Gamma+p)\left(\overline{\mathrm{I}}_{K} \cdot \overline{\mathrm{d}}_{\mathrm{K}}^{\mathrm{n}}\right)+\sum_{\mathrm{L}>\mathrm{K}} \overline{\mathrm{I}}_{\mathrm{K}} \cdot \overline{\mathrm{D}}_{\mathrm{KL}} \cdot \overline{\mathrm{I}}_{\mathrm{L}}\right\},
\end{aligned}
$$

$$
\mathrm{h}_{2}=\mathrm{H}_{1}+\mathrm{H}_{\mathrm{SR}}^{(e)}=
$$

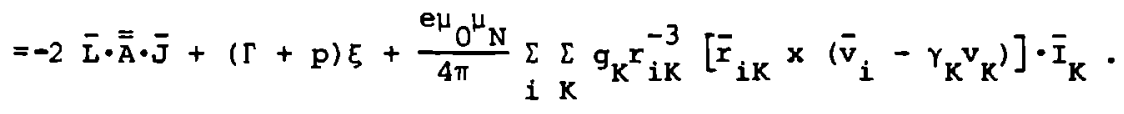

Herein $h_{0}$ represents the unperturbed Hamiltonian and $h_{1}$ terms of the perturbed Hamiltonian, which are diagonal with respect to the electronic state; $h_{2}$ contains also terms which are off-diagonal in the electronic states.

We perform now a perturbation calculation on $h_{2}$ up to second order with respect to the electronic state. The general formula is:

$$
\left\langle h_{2}\right\rangle=\left\langle 0\left|h_{2}\right| 0\right\rangle+\underset{n}{\Sigma^{\prime}}\left\langle 0\left|h_{2}\right| n>\left\langle n\left|h_{2}\right| 0\right\rangle\left(E_{0}-E_{n}\right)^{-1}\right.
$$

where $|0\rangle$ is the ground electronic state with energy $E_{0}$, and $|n\rangle$ the $n$-th excited state with energy $E_{n}$.

(a) First order.

For a ${ }^{1} \Sigma$ molecule $\left\langle 0\left|H_{1}\right| 0\right\rangle=0$ and consequently:

$$
\begin{aligned}
\left\langle 0\left|\mathrm{~h}_{2}\right| 0\right\rangle & =\left\langle 0\left|\mathrm{H}_{\mathrm{SR}}^{(e)}\right| 0\right\rangle= \\
& =\left\langle 0\left|\frac{e \mu_{0}^{\mu} \mathrm{N}}{4 \pi} \underset{1}{\sum} \sum_{\mathrm{K}} \mathrm{g}_{\mathrm{K}} \mathrm{K}_{\mathrm{iK}}^{-3}\left\{\overline{\mathbf{r}}_{1 \mathrm{~K}} \times\left(\overline{\mathrm{v}}_{1}-\gamma_{\mathrm{K}} \overline{\mathrm{v}}_{\mathrm{K}}\right)\right\} \cdot \overline{\mathrm{I}}_{\mathrm{K}}\right| 0\right\rangle
\end{aligned}
$$

If we approximate $\left.<0\left|x_{i K}^{-3}\left(\bar{r}_{i K} \times \bar{v}_{i}\right)\right| 0\right\rangle$ by $\left\langle 0\left|x_{i K}^{-3}\right| 0>\left\langle 0\left|\bar{r}_{i K} \times \bar{v}_{1}\right| 0\right\rangle\right.$ 
then this term is zero for ${ }^{1} \Sigma$ molecules. For $\bar{v}_{K}$ we use again Eq. (2) and $\left.<0\left|h_{2}\right| 0\right\rangle$ simplifies to:

$\left\langle 0\left|h_{2}\right| 0\right\rangle=\frac{-e \mu_{0}^{\mu} N}{4 \pi}\left\langle 0\left|\sum_{1} \sum_{K} \gamma_{K} g_{K} I_{i K}^{-3}\left\{\bar{I}_{i K} \times\left[\left(\bar{\omega} \times \bar{I}_{K}\right)-\left(\dot{\alpha} \times \bar{\sigma}_{K}\right)\right]\right\} \cdot \bar{I}_{K}\right| 0\right\rangle$

By substituting herein Eq. (16) we get:

$\left\langle 0\left|h_{2}\right| 0\right\rangle=\sum_{K} \bar{I}_{K} \cdot \overline{\bar{N}}_{K}^{e 1} \cdot \bar{J}+(\Gamma+p)\left(\bar{I}_{K} \cdot \bar{d}_{K}^{e 1}\right)$

with:

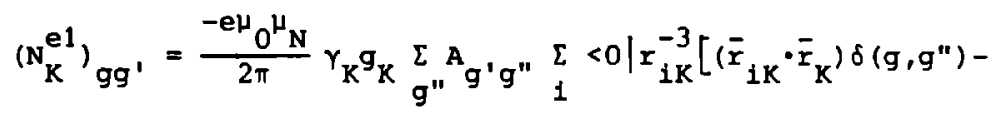

$$
\begin{aligned}
& \left.-\left(\bar{r}_{K}\right)_{g}\left(\bar{r}_{i K}\right)^{\prime \prime}\right]|O\rangle, \\
& \overline{\mathrm{d}}_{\mathrm{K}}^{(\mathrm{e} 1)}=\frac{-\mathrm{e} \mu_{0} \mu_{\mathrm{N}}}{2 \pi} \gamma_{\mathrm{K}} \mathrm{g}_{\mathrm{K}} \sum_{i} \mathrm{~F}<0\left|\overline{\mathrm{r}}_{\mathrm{iK}} \times\left\{\left(\bar{\rho} \times \bar{r}_{\mathrm{K}}\right)-\left(\bar{\lambda}^{\mathrm{N}} \bar{\sigma}_{\mathrm{K}}\right)\right\}\right| 0>.
\end{aligned}
$$

(b) Second order.

The second order contribution is a sum of the terms:

$$
\Sigma^{\prime}<0\left|h_{2}\right| n>\left\langle n\left|h_{2}\right| 0\right\rangle\left(E_{0}-E_{n}\right)^{-1}=U+v+w,
$$

where:

$$
\begin{aligned}
& U=\Sigma_{n}<<0\left|H_{1}\right| n><n\left|H_{1}\right| 0>\left(E_{0}-E_{n}\right)^{-1}, \\
& V=\Sigma_{n}^{\prime}\left\langle 0\left|H_{1}\right| n>\left\langle n\left|H_{S R}^{(e)}\right| 0\right\rangle\left(E_{0}-E_{n}\right)^{-1}+c . c .,\right. \\
& \mathbf{W}=\underset{n}{\Sigma} \cdot<0\left|H_{S R}^{(e)}\right| n><n\left|H_{S R}^{(e)}\right| 0>\left(E_{0}-E_{n}\right)^{-1} .
\end{aligned}
$$


We neglect the contribution of $\sum_{1} \gamma_{K} r_{i K}^{-3}\left(\bar{r}_{1 K} \times \bar{v}_{K}\right) \cdot \bar{I}_{K}$ to $H_{S R}^{(e)}$ because this term is already taken into account in first order. Then $H_{S R}^{(e)}$ simplifies to:

$$
\begin{aligned}
\mathrm{H}_{\mathrm{SR}}^{(e)} & =\frac{e \mu_{0} \mu_{N}}{4 \pi m} \sum_{1} \sum_{K} g_{K} r_{1 K}^{-3}\left(\bar{r}_{1 K} \times \bar{p}_{i}\right) \cdot \bar{I}_{K} \\
& =\frac{e \mu_{0} \mu_{N}}{4 \pi m} \sum_{K} g_{K}\left(\frac{\bar{L}^{\prime}}{r} \cdot \bar{I}_{K}\right),
\end{aligned}
$$

with:

where:

$$
\left(\frac{\bar{L}^{\prime}}{r^{3}}\right)=\sum_{i}\left(\bar{r}_{i K} \times \bar{p}_{i}\right) r_{i K}^{-3},
$$

$$
\bar{p}_{1}=m \bar{v}_{i} \text {, and } m \text { is the electron mass. }
$$

By substituting $H_{1}$ of Eq. (11b) into Eq.(21a) we obtain for U :

$$
\begin{aligned}
U & =\Sigma_{n}^{\prime}<0|-2 \bar{L} \cdot \overline{\bar{A}} \cdot \bar{J}+\xi(\Gamma+p)| n>\left\langle n|-2 \bar{L} \cdot \bar{A} \cdot \bar{J}+\xi(\Gamma+p)| 0>\left(E_{0}-E_{n}\right)^{-1}\right. \\
& =\bar{J} \cdot \overline{\bar{A}}(e) \cdot \bar{J}+\left(\bar{J} \cdot \bar{D}^{(e)}\right)(\Gamma+p)+F^{(e)}(\Gamma+p)^{2}
\end{aligned}
$$

with:

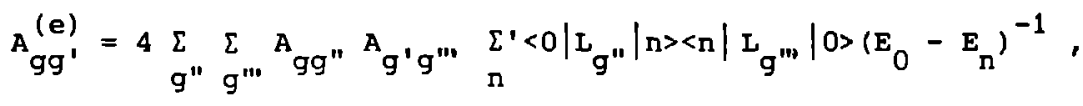

$$
\begin{aligned}
& \mathrm{D}_{g}^{(e)}=-2 \sum_{g^{\prime}} A_{g g^{\prime}} \sum_{n}^{\Sigma^{\prime}<0\left|L_{g^{\prime}}\right| n>\langle n|\xi| 0\rangle\left(E_{0}-E_{n^{\prime}}\right)^{-1}+c . c .} \text {, } \\
& F^{(e)}=\sum_{K}<0|\xi| n>\left\langle n|\xi| 0>\left(E_{0}-E_{n}\right)^{-1} .\right.
\end{aligned}
$$

It is readily seen from these expressions that $U$ can be absorbed in $\mathrm{H}_{0}$ of Eq. (11a) as can be shown by a rather lengthy elementary calculation. The resulting new $\mathrm{H}_{0}$ has the same form as Eq. (11a) with only slightly different values of $F$ and the components of $\bar{A}$ and $\bar{\rho}$ (or $\bar{\lambda}$ ). We neglect the term $U$ in the following.

The next second-order contribution is: 


$$
\begin{aligned}
V & =\Sigma_{\Omega}^{\prime}<0\left|H_{1}\right| n>\left\langle\Omega\left|H_{S R}^{(e)}\right| 0\right\rangle\left(E_{0}-E_{n}\right)^{-1}+c . c . \\
& =\frac{e{ }_{0}^{\mu} N}{4 \pi m} \sum_{K} \Sigma^{\prime}<0|-2 \bar{L} \cdot \overline{\bar{A}} \cdot \bar{J}+\xi(\Gamma+p)| n>\left\langle\Omega\left|g_{K}\left(\frac{\bar{L}^{\prime}}{I^{3}} \cdot \bar{I}_{K}\right)\right| 0\right\rangle+c . c . \\
& =\sum_{K} \bar{I}_{K} \cdot \overline{\bar{N}}_{K}^{e 2} \cdot \bar{J}+(\Gamma+p)\left(\bar{I}_{K} \cdot \bar{d}_{K}^{e 2}\right),
\end{aligned}
$$

with:

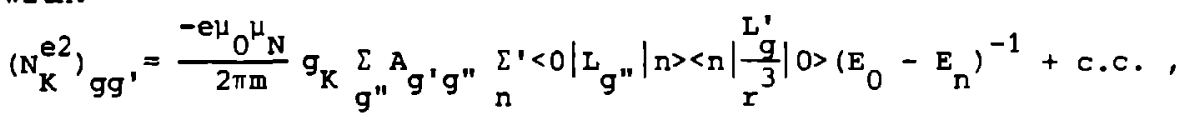

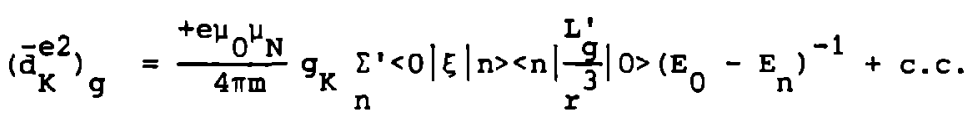

The last second-order contribution is:

$$
\begin{aligned}
& W=\Sigma_{n}^{\prime}<0\left|H_{S R}^{(e)}\right| n>\left\langle\Omega\left|H_{S R}^{(e)}\right| O>\left(E_{0}-E_{n}\right)^{-1}\right. \\
& =\left[\frac{{ }_{0} \mu_{N}}{4 \pi m}\right]^{2} \Sigma_{n}^{\prime}\left\langle 0\left|\sum_{K} g_{K}\left(\frac{\bar{L}^{\prime}}{I^{3}} \cdot \bar{I}_{K}\right)\right| n>\left\langle\pi\left|\sum_{L} g_{L}\left(\frac{\bar{L}^{\prime}}{r^{3}} \cdot \bar{I}_{L}\right)\right| 0\right\rangle\right. \\
& =\sum_{K, L} \bar{I}_{K} \cdot \overline{\bar{D}}_{K L}^{e} \cdot \bar{I}_{L},
\end{aligned}
$$

with:

$$
\left(D_{K L}^{e}\right)^{\prime} g g^{\prime}=\left[\frac{{ }^{\mu}{ }^{\mu} N_{N}}{4 \pi m}\right]^{2} g_{K^{\prime}} g_{n} \Sigma^{\prime}<0\left|\frac{L^{\prime}}{g^{3}}\right| n><n\left|\frac{L^{\prime}}{g^{\prime}}\right| O>\left(E_{0}-E_{n^{\prime}}\right)^{-1}+c . c .
$$

This term 1 s the so called indirect or electron-coupled spin-spin interaction. It can be neglected in most cases.

By comblning Eqs. (17), (20), (24), and (2:5) we obtain:

$$
\mathrm{H}_{\mathrm{HF}}=\sum_{\mathrm{K}}\left\{\overline{\mathrm{I}}_{\mathrm{K}} \cdot \overline{\overline{\mathrm{N}}}_{\mathrm{K}} \cdot \overline{\mathrm{J}}+(\Gamma+\mathrm{p})\left(\overline{\mathrm{I}}_{\mathrm{K}} \cdot \overline{\mathrm{d}}_{\mathrm{K}}\right)+\sum_{\mathrm{L}>\mathrm{K}} \overline{\mathrm{I}}_{\mathrm{K}} \cdot \overline{\overline{\mathrm{D}}}_{\mathrm{KL}} \cdot \overline{\mathrm{I}}_{\mathrm{L}}\right\},
$$

where:

$$
\overline{\overline{\mathrm{N}}}_{K}=\overline{\overline{\mathrm{N}}}_{K}^{\mathrm{n}}+\overline{\overline{\mathrm{N}}}_{\mathrm{K}}^{\mathrm{e} 1}+\overline{\overline{\mathrm{N}}}_{\mathrm{K}}^{\mathrm{e} 2} \text {, }
$$




$$
\overline{\mathrm{d}}_{\mathrm{K}}=\overline{\mathrm{d}}_{\mathrm{K}}^{\mathrm{n}}+\overline{\mathrm{d}}_{\mathrm{K}}^{\mathrm{e} 1}+\overline{\mathrm{d}}_{\mathrm{K}}^{\mathrm{e} 2} \text {. }
$$

The first term of $\mathrm{H}_{\mathrm{HF}}$ is the normal spin-rotation interaction, which is the same as in rigid rotor molecules. The second term is the spin-internal rotation interaction, which vanishes if $p \rightarrow 0$ and $\bar{\lambda} \rightarrow 0$. The last term is the spin-spin interaction.

The hyperfine Hamiltonian can be written in a different form, which is more convenient for calculating the matrix elements:

$$
\mathrm{H}_{\mathrm{HF}}=\sum_{\mathrm{K}}\left\{\overline{\mathrm{I}}_{\mathrm{K}} \cdot \overline{\overline{\mathrm{M}}}_{\mathrm{K}} \cdot \overline{\mathrm{J}}+\mathrm{P}\left(\overline{\mathrm{I}}_{\mathrm{K}} \cdot \overline{\mathrm{d}}_{\mathrm{K}}\right)+\sum_{\mathrm{L}>\mathrm{K}} \overline{\mathrm{I}}_{\mathrm{K}} \cdot \overline{\overline{\mathrm{D}}}_{\mathrm{KL}} \cdot \overline{\mathrm{I}}_{\mathrm{L}}\right\},
$$

with:

$$
\left(M_{K}\right)^{\prime} g g^{\prime}=\left(N_{K^{\prime}}\right)^{\prime} g^{\prime}+\rho_{g}\left(d_{K^{\prime}}\right)^{\prime} \cdot
$$

The Hermitian form of this Hamiltonian is:

$$
\begin{aligned}
\mathrm{H}_{\mathrm{HF}}=\sum_{\mathrm{K}}\left\{\frac{1}{2}\left(\overline{\mathrm{I}}_{\mathrm{K}} \cdot \overline{\bar{M}}_{\mathrm{K}} \cdot \overline{\mathrm{J}}\right)+\right. & \frac{1}{2}\left(\overline{\mathrm{I}}_{\mathrm{K}} \cdot \overline{\overline{\mathrm{M}}}_{\mathrm{K}} \cdot \overline{\mathrm{J}}\right)^{\dagger}+\frac{1}{2}\left(\overline{\mathrm{I}}_{\mathrm{K}} \cdot \mathrm{p} \overline{\mathrm{d}}_{\mathrm{K}}\right)+ \\
& \left.+\frac{1}{2}\left(\overline{\mathrm{I}}_{\mathrm{K}} \cdot \mathrm{p}_{\mathrm{K}}\right)^{\dagger}+\sum_{\mathrm{L}>\mathrm{K}} \overline{\mathrm{I}}_{\mathrm{K}} \cdot \overline{\overline{\mathrm{D}}}_{\mathrm{KL}} \cdot \overline{\mathrm{I}}_{\mathrm{L}}\right\} .
\end{aligned}
$$

II. METHANOL-LIKE MOLECULES.

The preceeding treatment is subject to only one fundamental restriction, that the molecule has only one internal degree of freedom (a). Now we treat the more specific case of a molecule consisting of two parts, one part called the "top", is a symetrical top and the other part, called the "frame", has a plane of symmetry, which contains the symmetry axis of the top. The top can rotate with respect to the frame about the symmetry axis with angular velocity $\dot{\alpha}\left(\mathrm{CH}_{3} \mathrm{OH}\right.$ for example). We choose a rlght-handed coor- 
dinate system fixed in the frame with the origin in the center of mass of the whole molecule. The z-axis of this system is parallel to the symmetry axis of the top, the $x$-axis is perpendicular to the plane of symmetry of the frame and the y-axis, lying in this plane, is perpendicular to both (Fig. 1, Appendix).

If $A, B$ and $C$ are the moments of inertia about the $x, y$ and $z-$ axis, respectively, $-D$ the product of iriertia $I_{y z}\left(I_{x y}=I_{x z}=0\right)$, $c_{2}$ the moment of inertia of the top about 1 ts symetry axis, and

$$
\begin{gathered}
\left.C_{1}=c-C_{2}=A-B \text { we can write (see also }(\underline{5}, \underline{6})\right) \text { for } H_{0} \text { (Eq } \\
\begin{aligned}
H_{0}=\frac{1}{2 A} J_{x}^{2} & +\frac{1}{2\left(B C_{1}-c^{2}\right)}\left\{C_{1} J_{y}^{2}+B J_{z}^{2}+D\left(J_{y} J_{z}+J_{z} J_{Y}\right)+\right. \\
& \left.+2 D\left(p J_{y}\right)+2 B\left(p J_{z}\right)+\frac{B C-D^{2}}{C_{2}} p^{2}\right\}+V(\alpha)
\end{aligned}
\end{gathered}
$$

Explicit expressions for the components of $\bar{J}$ in the present frame of reference are given in the Appendix.

In the following we restrict ourselves to molecules with a threefold potential barrier $V(\alpha)$ and in particular to molecules whose top is formed by the methyl radical $\mathrm{CH}_{3}$. There are two problems that can be solved with great advantage using grouptheoretical methods: (1) calculation of the elgenfunctions and eigenvalues of $\mathrm{H}_{0}$ with an appropriate set of basis functions, and (2) determination of the wavefunctions, including the nuclear spin wavefunctions, which do not violate the Pauli-Exclusion Principle.

\section{The molecular symmetry group.}

It is readily shown that the Hamiltonian $\mathrm{H}_{0}$ given in Eq. (28) is invariant under the following basic operations (and their pro- 
ducts) of the internal symmetry group (7) : $E$ (Identity), $\mathrm{c}_{3}$ and $\mathrm{c}_{2 \mathrm{x}}$. Under these operations the components of $\bar{J}, p$ and $\alpha$ transform as:

$$
\begin{aligned}
& E: J_{x, Y, Z} \rightarrow J_{x, y, z}: p \rightarrow p, \alpha \rightarrow \alpha \\
& C_{3}: J_{x, y, z} \rightarrow J_{x, y, z}: p \rightarrow p, \alpha \rightarrow \alpha+\frac{2 \pi}{3} \\
& c_{2 x}: J_{x} \rightarrow J_{x}: J_{y, z} \rightarrow-J_{y, z} ; p \rightarrow-p, \alpha \rightarrow-\alpha
\end{aligned}
$$

These operations can be expressed in terms of the Euler angles $\psi, \theta$ and $\phi_{1}$, and the relative angle $a$ :

$$
\begin{aligned}
& \mathrm{E}: \psi \rightarrow \psi \quad: \theta \rightarrow \theta \quad: \phi_{1} \rightarrow \phi_{1} \quad ; \alpha+\alpha \\
& \mathrm{C}_{3}: \psi \rightarrow \psi \quad ; \phi_{1} \rightarrow \phi_{1} \quad ; \alpha \rightarrow \alpha+\frac{2 \pi}{3} \\
& \mathrm{C}_{2 \mathrm{x}}: \psi \rightarrow \psi+\pi: \theta \rightarrow \pi-\theta ; \phi_{1} \rightarrow 2 \pi-\phi_{1} ; \alpha+-\alpha
\end{aligned}
$$

They constitute a group, which is isomorphic with the group $\mathrm{C}_{3 \mathrm{v}}$ with irreducible representations: $A_{1}, A_{2}$ and $E$.

The eigenfunctions of $H_{0}$ have the symmetry of the species $A_{1}$, $A_{2}$ or $E$. A method to obtain these eigenfunctions is given in the Appendix. The electric dipole moment of this type of molecules has the symmetry properties of $A_{2}$, because it lles in the symmetry plane of the frame. The selection rules for the electric dipole transitions are easily derived from the Wigner-Eckart theorem as:

$$
A_{1} \leftrightarrow A_{2} \text { and } E \leftrightarrow E
$$

Longuet-Higgins (B) determines the symetry operations of the molecular group in a different way, which is more suitable for the nuclear spin wavefunctions. Let $P$ be any permutation of the positi- 
ons and spins of ldentical nuclei, or any product of such permutations. If $\mathrm{E}$ is the identity operation and $\mathrm{E}^{\mathbf{H}}$ the operator inverting the positions of all particles in the center of mass, then the molecular symmetry group is a set of:

1) all feasible $P$, including $E$,

11) all feasible products $P E^{X}=E^{X} P$, where "feasible" means leaving the Hamiltonian 1nvariant. The operation $E^{M}\left(J_{x} \rightarrow-J_{x}, J_{y} \rightarrow-J_{y}, J_{z} \rightarrow-J_{z} ; \alpha+\alpha\right)$ and pèrmutations which mexely exchange two protons in the $\mathrm{CH}_{3}$-group $\left(\mathrm{J}_{\mathbf{x}} \rightarrow \mathrm{J}_{\mathbf{x}}, \mathrm{J}_{\mathbf{y}}+\mathrm{J}_{\mathbf{y}}\right.$ ' $\left.J_{\mathbf{z}} \rightarrow J_{z} ; \alpha \rightarrow-\alpha\right)$ do not satisfy the criterion of feasibility. The remaining operations, whlch do satisfy the requirements (i) and (11) are:

1) 1dentity $\mathrm{E}$

2) cyclic permutations of the three protons in the methyl rad1$\operatorname{cal}\left(\mathrm{C}_{3}\right.$ and $\mathrm{C}_{3}{ }^{2}$ )

3) permutations of only two protons followed by the inversion $E^{x}\left(C_{2 x}, C_{2 x} C_{3}, C_{2 x} c_{3}^{2}\right)$

In the case of three equivalent protons, there are six independent spin wavefunctions, linear combinations of triple products of $\alpha_{i}$ and $\beta_{i}$, where $\alpha_{i}, \beta_{i}$ stands for the spin wavefunction of the 1 -th proton with spin up or down, respectively. This set generates a reducible representation of the group. The problem of finding the basis sets for the irreducible representations of the group $\mathrm{C}_{3 \mathrm{v}}$ reduces to the problem of finding them for the group $s_{3}$, since all spln wavefunctions are invariant under the inversion $\mathrm{E}^{\mathrm{x}}$. This problem has been solved by Gunther-Mohr et.al. (1) and the result is reproduced in Table $I$. The spin wavefunctions are given in the 
$\mid\left(I_{1} I_{2}\right) I_{12} I_{3} I M_{I}>$ representation, which corresponds to the coupling scheme:

$$
\begin{aligned}
& \bar{I}_{1}+\bar{I}_{2}=\bar{I}_{12}, \\
& \bar{I}_{12}+\bar{I}_{3}=\bar{I},
\end{aligned}
$$

where $\bar{I}_{1}, \bar{I}_{2}$ and $\bar{I}_{3}$ are the spins of the three protons of the $\mathrm{CH}_{3}-$ group. The nuclear spins in the frame are taken into account lateron.

Table I. The spin basis functions of the irreducible representations of the group $C_{3 v}$.

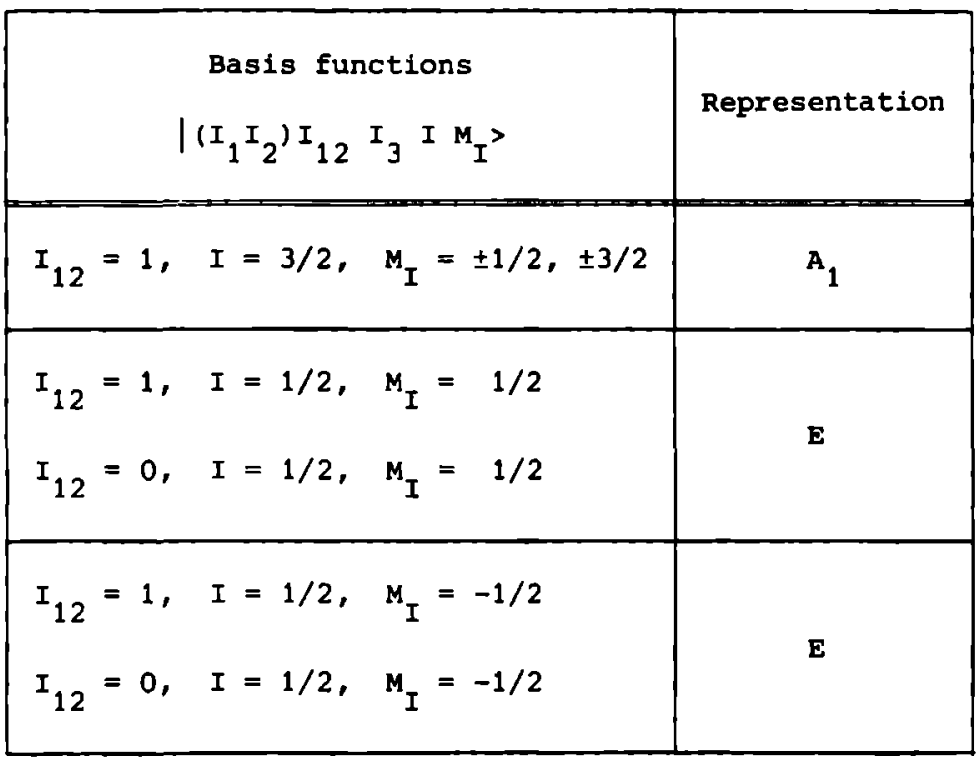

In order to calculate the hyperfine structure of a certain rotational level, we have to determine a set of appropriate total wavefunctions, including nuclear spins, which satisfy the following condtions : 
1) form a basis set for an Irreducible representation of the molecular symmetry group.

i1) are not forbldden by the Pauli Exclusion Principle. According to the last requirement, the total wavefunctions should be antisymmetric for exchange of two 1dentical protons, and hence symmetric for a cyclic permutation of the three protons in the methyl radical (class $2 \mathrm{C}_{3}$ ). The exclusion principle is not committal for the operations $\mathrm{PE}^{\mathrm{x}}$ belonging to the class $\left(3 \mathrm{C}_{2 \mathrm{x}}\right)$, because they do not merely permute identical protons. We conclude that the total wavefunction should be of species $A_{1}$ or $A_{2}$ and that total wavefunctions of spectes $\mathrm{E}$ are forbidden.

The rotational levels are classified as species $\mathrm{A}_{1}, \mathrm{~A}_{2}$ or $\mathrm{E}$. In the Appendix is shown that levels of species A can be denoted by $\left(J_{\gamma^{+}}\right)$or $\left(J_{\gamma_{-}}\right)$with eigenfunctions $\left|J_{\gamma^{+}} M_{J^{\prime}}\right\rangle$ or $\left|J_{\gamma^{-}} M_{J^{\prime}}\right\rangle$, respectively. The doubly degenerate levels of species $E$ are denoted by $\left(J_{Y^{\prime}}\right)$ with eigenfunctions $\left|J_{\gamma^{\prime}+} M_{J^{\prime}}\right\rangle$ and $\left|J_{Y^{\prime}-} M_{J^{\prime}}\right\rangle$. The symbol $\gamma\left(\gamma^{\prime}\right)$ labels the rotational states with the same $J$. The transformation of the wavefunctions under the operation $c_{2 x}$ is given by: $c_{2 x}\left|J_{\gamma \pm} M_{J}\right\rangle= \pm(-1)^{J}\left|J_{Y \pm} M_{J}\right\rangle$, both for functions of $A$ and $E$ species.

For the spin wavefunctions as given in Table I we use the shorthand notation $\left|I_{12} I M_{I}\right\rangle$ for $\left|\left(I_{1} I_{2}\right) I_{12} I_{3} I M_{I}\right\rangle$. The set of functions, which are products of the rotational eigenfunctions $\left|J_{\gamma^{ \pm}} M_{J}\right\rangle$ and the spin wavefunctions $\left|I_{12} I M_{I}\right\rangle$ form a basis for a reducible representation of the symmetry group. This representation can be reduced by taking the proper linear combinations of these product functions. The result is given in Table II. Forbidden total 
Table II. The total basis functions and their symetry belonging to the rotational states.

\begin{tabular}{|c|c|c|c|c|c|c|}
\hline \multicolumn{4}{|c|}{ ROTATIONAL } & \multicolumn{3}{|l|}{ TOTAL } \\
\hline \multirow{2}{*}{ State } & \multicolumn{2}{|c|}{ Symmetry } & \multirow{2}{*}{ Wavefunction } & \multirow{2}{*}{ Basis functions ${ }^{a}$} & \multicolumn{2}{|c|}{ Symmetry } \\
\hline & J-even & $J-o d d$ & & & J-even & J-odd \\
\hline$\left.{ }^{\left(J_{\gamma+}\right.}\right)$ & $A_{1}$ & $A_{2}$ & $\left|J_{\gamma+} M_{J}\right\rangle$ & $\left|J_{\gamma+} M_{J}>\right| 1 \frac{3}{2} M_{I}>$ & $A_{1}$ & $\mathrm{~A}_{2}$ \\
\hline$\left(J_{\gamma-}\right)$ & $A_{2}$ & $A_{1}$ & $\left|\mathrm{~J}_{\gamma-} \mathrm{M}_{\mathrm{J}}\right\rangle$ & $\left|J_{Y-} M_{J}\right\rangle \mid \begin{array}{lll}1 & \frac{3}{2} & M_{I}\end{array}$ & $A_{2}$ & $\mathrm{~A}_{1}$ \\
\hline$\left(\mathrm{J}_{\gamma}\right)$ & $\mathbf{E}$ & $\mathbf{E}$ & $\left|J_{Y-{ }_{J}}\right\rangle$ & $\begin{array}{l}\frac{1}{\sqrt{2}}\left\{\left|J_{\gamma-{ }_{J}}>\right| 0 \frac{1}{2} M_{I}>+1\left|J_{\gamma+} M_{J}>\right| 1 \frac{1}{2} M_{I}>\right\} \\
\frac{1}{\sqrt{2}}\left\{\left|J_{\gamma+} M_{J}>\right| 0 \frac{1}{2} M_{I}>+1\left|J_{\gamma-} M_{J}>\right| 1 \frac{1}{2} M_{I}>\right\}\end{array}$ & $\begin{array}{l}\mathrm{A}_{1} \\
\mathrm{~A}_{2}\end{array}$ & $A_{2}$ \\
\hline
\end{tabular}

ancluding the three proton spins in the top, but excluding other spins in the top or frame. 
wavefunctions of species $E$ are omitted.

The basis functions of Table II have to be extended with the spin wavefunctions $\left|I_{N} M_{N}\right\rangle$ of the nuclear spins $\bar{I}_{N}$ in the frame of the molecule. The basis functions in Table II, and hence also the extended total wavefunctions, are given in the uncoupled representation $\left|M_{J}, M_{I}, M_{N}\right\rangle$. In the absence of an external electric or magnetic fleld, however, it is more conventent to calculate the matrix elements of the Hamiltonian in the coupling scheme, in which the angular momenta $\bar{J}, \bar{I}$ and $\bar{I}_{N}$ are all coupled to the total angular momentum $\bar{F}$, since $F$ and $M_{F}$ are good quantum numbers in contrast to $\mathrm{M}_{\mathrm{J}}, \mathrm{M}_{\mathrm{I}}$ and $\mathrm{M}_{\mathrm{N}}$. The wavefunctions corresponding to this coupling scheme (Eqs. (33) and (34)) are merely linear combinations of the uncoupled wavefunctions given in Table II and so have the same symmetry propertles. From the Wigner-Eckart theorem it follows that all matrix elements between basis functions of different species vanish. The rotational part $\mathrm{H}_{0}$ of the Hamiltonian is, of course, diagonal In all quantum numbers, but the hyperfine Hamiltonian $\mathrm{H}_{\mathrm{HF}}$ is not. Since in general the hyperfine interactions are much weaker than molecular rotation, matrix elements of $\mathrm{H}_{\mathrm{HF}}$ off-diagonal in the rotational quantum numbers $J$ and $\gamma_{( \pm)}$can be neglected. If the rotational splittings are very small (K-doublets) or zero (E-levels), these matrix elements vanish because of the symmetry. The Hamiltonian matrlx splits into a number of submatrices that can be characterized by $J$ and $Y$ and symmetry type $A_{1}$ or $A_{2}$. The two submatrices of species $A_{1}$ and $A_{2}$, which belong to one particular rotational level $\left(J_{\gamma}\right)$ of species $E$, are identical. This implies that the degeneracy is not lifted by the hyperfine interactions. 
III. THE HYPERFINE STRUCTURE.

A. THE MATRIX ELEMENTS.

The most appropriate coordinate system for the calculation of the matrix elements of $\mathrm{H}_{\mathrm{HF}}(\mathrm{Eg} \cdot(28))$ is the space fixed CM-system $(X, Y, Z)$. For this reason the Hamiltonian is transformed from the molecule fixed $(x, y, z)$ to the space fixed $(X, Y, Z)$ system. In order to calculate the matrix elements with tensor operator techniques (9) all operators of the hyperfine Hamiltonian are expressed in spherical tensor operators:

$$
\begin{aligned}
& \overline{\mathrm{I}}_{\mathrm{K}} \cdot \overline{\bar{M}}_{\mathrm{K}} \cdot \overline{\mathrm{J}} \quad=\sum_{\nu=0,1,2}(-)^{\nu}(2 \nu+1)^{\frac{1}{2}}\left\{I_{\mathrm{K}}^{(1)}\left\{\mathrm{M}_{\mathrm{K}}^{(\nu)} \mathrm{J}^{(1)}\right\}^{(1)}\right\}^{(0)}, \\
& {\left[\bar{I}_{K} \cdot \overline{\bar{M}}_{K} \cdot \bar{J}\right]^{\dagger}=\sum_{v=0,1,2} \quad(2 v+1)^{\frac{1}{2}}\left\{\left\{J^{(1)}{ }_{M_{K}}^{(v)}\right\}^{(1)} I_{K}^{(1)}\right\}(0),} \\
& p\left(\bar{I}_{K} \cdot \bar{d}_{K}\right)=-\sqrt{3}\left\{I_{K}^{(1)}\left\{p_{p}^{(0)} d_{K}^{(1)}\right\}{ }^{(1)}\right\}{ }^{(0)} \text {. } \\
& {\left[\mathrm{p}\left(\overline{\mathrm{I}}_{\mathrm{K}} \cdot \overline{\mathrm{d}}_{\mathrm{K}}\right)\right]^{\dagger}=-\sqrt{3}\left\{\left\{\mathrm{~d}_{\mathrm{K}}^{(1)} \mathrm{P}^{(0)}\right\}^{(1)} \mathrm{I}_{\mathrm{K}}^{(1)}\right\}{ }^{(0)} \text {, }} \\
& \overline{\mathrm{I}}_{\mathrm{K}} \cdot \overline{\bar{D}}_{\mathrm{KL}} \cdot \overline{\mathrm{I}}_{\mathrm{L}}=\sqrt{5}\left\{\mathrm{D}_{\mathrm{KL}}^{(2)}\left\{I_{\mathrm{K}}^{(1)} \mathrm{I}_{\mathrm{L}}^{(1)}\right\}^{(2)}\right\}^{(0)} \\
& =\sqrt{5}\left\{I_{K}^{(1)}\left\{D_{K L}^{(2)} I_{L}^{(1)}\right\}^{(1)}\right\}{ }^{(0)} \text {. }
\end{aligned}
$$

with $(\underline{10})$ :

$$
\begin{aligned}
& \mathbf{p}_{0}^{(0)}=\mathbf{p} \quad, \\
& v_{ \pm 1}^{(1)}=\mp\left(\frac{1}{2}\right)^{\frac{1}{2}}\left(v_{x} \pm 1 v_{Y}\right), \\
& v_{0}^{(1)}=v_{z},
\end{aligned}
$$

*) $p$ is really a tensor of rank zero since it commutes with $J_{X}, J_{Y}$, and $\mathrm{J}_{\mathbf{Z}}$ 


$$
\begin{aligned}
& T_{0}^{(0)}=-\left(\frac{1}{3}\right)^{\frac{1}{2}}\left(T_{X X}+T_{Y Y}+T_{Z Z}\right), \\
& T_{ \pm 1}^{(1)}=\mp \frac{1}{2}\left[\left(T_{Y Z}-T_{Z Y}\right) \mp i\left(T_{Z X}-T_{X Z}\right)\right], \\
& T_{0}^{(1)}=\left(\frac{1}{2}\right)^{\frac{1}{2}} 1\left(T_{X Y}-T_{Y X}\right), \\
& T_{ \pm 2}^{(2)}=\frac{1}{2}\left[\left(T_{X X}-T_{Y Y}\right) \pm 1\left(T_{X Y}+T_{Y X}\right)\right], \\
& T_{ \pm 1}^{(2)}=\frac{1}{2}\left[\left[\left(T_{X Z}+T_{Z X}\right)-i\left(T_{Y Z}+T_{Z Y}\right)\right],\right. \\
& T_{0}^{(2)}=\left(\frac{1}{6}\right)^{\frac{1}{2}}\left(2 T_{Z Z}-T_{X X}-T_{Y Y}\right),
\end{aligned}
$$

where $\overline{\mathrm{v}}$ stands for $\overline{\mathrm{J}}, \overline{\mathrm{I}}_{\mathrm{K}}$ or $\overline{\mathrm{d}}_{\mathrm{K}}$, and $\overline{\mathrm{T}}_{\text {for }} \overline{\overline{\mathrm{M}}}_{\mathrm{K}}$ or $\overline{\mathrm{D}}_{\mathrm{KL}}$.

The spherical tensor operators $D_{K L}{ }^{(0)}$ and $D_{K L}{ }^{(1)}$ are zero since $D_{K L}$ is a traceless and symetric Cartesian tensor.

The hyperfine matrix elements for a molecule with three equivalent protons (spins $\bar{I}_{1}, \bar{I}_{2}$ and $\bar{I}_{3}$ ) in the top and one nuclear spin $\overline{\mathrm{I}}_{4}$ located in the frame have been calculated, using the coupling scheme:

$$
\overline{\mathrm{J}}+\overline{\mathrm{I}}_{4}=\overline{\mathrm{F}}_{1}, \quad \overline{\mathrm{I}}_{1}+\overline{\mathrm{I}}_{2}=\overline{\mathrm{I}}_{12}, \quad \overline{\mathrm{I}}_{12}+\overline{\mathrm{I}}_{3}=\overline{\mathrm{I}}, \quad \overline{\mathrm{F}}_{1}+\overline{\mathrm{I}}=\overline{\mathrm{F}} .
$$

The corresponding set of basis functions for the rotational levels $\left(J_{\gamma \pm}\right)$ of species $A_{1}$ or $A_{2}$ are:

$$
\phi_{ \pm}=\left|\left(J_{\gamma \pm} I_{4}\right) F_{1},\left(\left(I_{1} I_{2}\right) I_{12}=1, I_{3}\right) I=\frac{3}{2}, F, M_{F}\right\rangle,
$$

and for the rotational levels $\left(\mathrm{J}_{\gamma}\right)$ of species $\mathrm{E}$ :

$$
\begin{aligned}
\psi_{ \pm}=\left(\frac{1}{2}\right)^{\frac{1}{2}}\left\{\mid\left(J_{Y_{\mp}} I_{4}\right) F_{1},\left(\left(I_{1} I_{2}\right) I_{12}=0, I_{3}\right) I=\frac{1}{2}, F, M_{F}>+\right. \\
\left.+1 \mid\left(J_{Y \pm 4} I_{4}\right) F_{1},\left(\left(I_{1} I_{2}\right) I_{12}=1, I_{3}\right) I=\frac{1}{2}, F, M_{F}>\right\} .
\end{aligned}
$$


The basis functions $\phi_{ \pm}$and $\psi_{ \pm}$are constructed out of the (uncoupled) total basis functions of Table II and the spin wavefunctions $\left|I_{4} M_{4}\right\rangle$ of the frame using standard techniques. It should be noted that to a particular rotational level of species $A$ belongs the set of functions $\phi_{+}$or $\phi_{-}$but not both sets together, while to a particular rotational level of species $\mathrm{E}$ belong both sets of functions $\psi_{+}$as well as $\psi_{-}$. The symmetry of the functions $\phi_{+}$or $\phi_{-}$is that of the level to which they belong. The basis functions $\psi_{+}$have symetry $A_{1}$ or $A_{2}$ and $\psi_{-}$have symmetry $A_{2}$ or $A_{1}$ for $J$ is even or odd, respectively, (Table II).

Defining $\phi_{ \pm}^{\prime}$ and $\psi_{ \pm}^{\prime}$ as the functions $\phi_{ \pm}$and $\psi_{ \pm}$with $F_{1}, F, M_{F}$ replaced by $F_{1}^{\prime}, F^{\prime}, M_{F}^{\prime}$, respectively, we can write for the matrix elements:

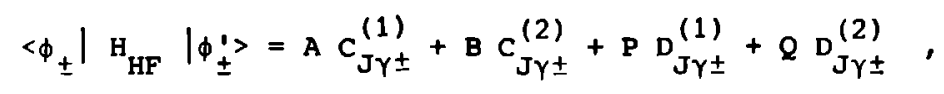

for the A-levels, and:

$$
\begin{aligned}
\left\langle\psi_{+}\left|\mathrm{H}_{\mathrm{HF}}\right| \psi_{+}^{\prime}\right\rangle & =\left\langle\psi_{-}\left|\mathrm{H}_{\mathrm{HF}}\right| \psi_{-}^{\prime}\right\rangle \\
& =A C_{\mathrm{J} Y}^{(1)}+\mathrm{B} \mathrm{C}_{\mathrm{J} Y}^{(2)}+\mathrm{Q} \mathrm{D}_{\mathrm{J} Y}^{(2)},
\end{aligned}
$$

for the E-levels.

The matrix elements $\left\langle\psi_{+}\left|\mathrm{H}_{\mathrm{HF}}\right| \psi_{-}^{\prime}\right\rangle$ are zero, because the wavefunctions $\psi_{+}$and $\psi_{-}$have different symetry. The terms in expression (36), which are mixed in $I$ ( $I=0$ and $I=1$ ) cancel; this can be proved using the hermiticity of the Hamiltonian.

The values of the coupling constants $C^{(i)}$ and $D^{(i)}$ depend on the rotational levels in question. They are labeled in the same way as the rotational levels $\left(J_{\gamma_{+}}\right.$or $J_{\gamma_{-}}$for $A-l e v e l s$, and $J_{\gamma}$ for 
E-levels) to which they belong. The constant $c^{(1)}$ refers to the spin-rotation interaction of the three protons in the top;

$c^{(2)}$ is the same for the nuclear spin $\bar{I}_{4}$ in the frame; $D^{(1)}$ is the coupling constant for the spin-spin interactlons between the protons in the top, and $\mathrm{D}^{(2)}$ is the coupling constant for the same interaction between the spin in the frame and the three proton spins in the top. The four coupling constants of an A-level are:

$$
\begin{aligned}
& \mathrm{C}_{\mathrm{J} Y \pm}^{(1)}=\sum_{\mathrm{K}=1}^{3}\left[\mathrm{CM}_{\mathrm{K}, \mathrm{J} Y \pm}+\mathrm{Cd}_{\mathrm{K}, \mathrm{J}{ }^{\prime} \pm}\right] \text {, } \\
& \mathrm{c}_{\mathrm{J}{ }^{ \pm}}^{(2)}=\mathrm{CM}_{4, \mathrm{~J}{ }^{ \pm} \pm}+\mathrm{Cd}_{4, \mathrm{~J}{ }^{ \pm} \pm} \\
& D_{J \gamma \pm}^{(1)}=s_{12, J Y \pm}+s_{13, J \gamma \pm}+s_{23, J Y \pm}, \\
& D_{J \gamma \pm}^{(2)}=s_{14, J \gamma \pm}+s_{24, J \gamma \pm}+s_{34, J \gamma \pm},
\end{aligned}
$$

and the three coupling constants for an E-level:

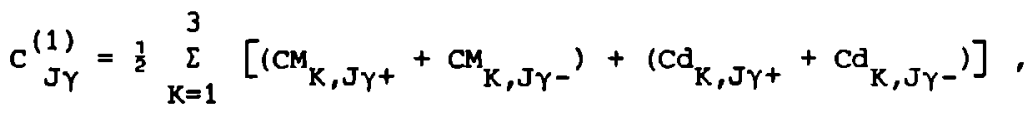

$$
\begin{aligned}
& \mathrm{C}_{\mathrm{J} \gamma^{(2)}}^{(2)}\left[\left(\mathrm{CM}_{4, \mathrm{~J}+}+\mathrm{CM}_{4, \mathrm{~J}_{\gamma^{-}}}\right)+\left(\mathrm{Cd}_{4, \mathrm{~J}^{+}}+\mathrm{Cd}_{4, \mathrm{~J}-}\right)\right] \text {, } \\
& \mathrm{D}_{\mathrm{J} \gamma}^{(2)}=\frac{1}{2} \sum_{\mathrm{K}=1}^{3}\left[\mathrm{~s}_{\mathrm{K} 4, \mathrm{~J} \gamma^{+}}+\mathrm{s}_{\mathrm{K} 4, \mathrm{~J} \gamma^{-}}\right] \text {. }
\end{aligned}
$$

The quantities $\mathrm{CM}_{\mathrm{K}}, \mathrm{Cd}_{\mathrm{K}}$ and $\mathrm{s}_{\mathrm{KL}}$ depend on the rotational wavefunction belonglng to the rotational level in question. They carry as subscript the quantum numbers of this wavefunction. Explicit expressions for these quantities are: 
$\mathrm{CM}_{K, J \gamma \pm}=\sum_{\nu=0,2}(2 \nu+1)^{\frac{1}{2}}\left\{\begin{array}{lll}1 & 1 & \nu \\ J & J & J\end{array}\left|<J_{\gamma \pm}\right| M_{K}^{(\nu)}\left|J_{\gamma \pm}\right\rangle\right.$,

$C d_{K, J \gamma \pm}=-\frac{1}{2}[J(J+1)(2 J+1)]^{-\frac{1}{2}}\left[\left\langle J{ }_{\gamma \pm}||\left\{P(0) d_{K}^{(1)}\right\}^{(1)} \mid J_{\gamma \pm}\right\rangle+\right.$

$+<J_{\gamma \pm}||\left\{d_{K}^{(1)} p^{(0)}\right\}^{(1)} \mid J_{\gamma \pm}>$,

$S_{K L, J Y \pm}=\frac{2}{3}\left[\frac{3 J(2 J-1)}{(2 J+1)(2 J+2)(2 J+3)}\right]^{\frac{1}{2}}\left\langle J_{\gamma \pm}\left\|D_{K L}(2)\right\|_{\gamma \pm}\right\rangle$.

The quantities A, B, P and $Q$ used in Eqs. (35) and (36) are given by:

$$
\begin{aligned}
& A=(-)^{J+2 F_{1}^{\prime}+F+I_{4}+I+1}\left[V \frac{5}{3} \delta\left(I, \frac{3}{2}\right)+\sqrt{ } \frac{1}{6} \delta\left(I, \frac{1}{2}\right)\right]\left[\left(2 F_{1}+1\right)\left(2 F_{1}^{\prime}+1\right) J(J+1)(2 J+1)\right]^{\frac{1}{2}} \\
& \left.\times\left(\begin{array}{ccc}
F_{1} & F_{1}^{\prime} & 1 \\
J & J & I_{4}
\end{array}\right\} \mid \begin{array}{ccc}
F_{1} & F_{1}^{\prime} & 1 \\
I & I & F
\end{array}\right\} \delta\left(F, F^{\prime}\right) \delta\left(M_{F}, M_{F}^{\prime}\right) \\
& B=\frac{1}{2}\left[F_{1}\left(F_{1}+1\right)-I_{4}\left(I_{4}+1\right)-J(J+1)\right] \delta\left(F_{1}, F_{1}^{\prime}\right) \delta\left(F, F^{\prime}\right) \delta\left(M_{F}, M_{F}^{\prime}\right) \text {, } \\
& P=(-)^{J+2 F_{1}^{\prime}+F+I_{4}+I} \frac{1}{2} \sqrt{5}\left[\frac{\left(2 F_{1}+1\right)\left(2 F_{1}^{\prime}+1\right)(2 J+1)(2 J+2)(2 J+3)}{2 J(J-1)}\right]^{\frac{1}{2}} \times \\
& \left.\times\left\{\begin{array}{lll}
F_{1} & F_{1}^{\prime} & 2 \\
J & J & I_{4}
\end{array}\right\} \mid \begin{array}{ccc}
F_{1} & F_{1}^{\prime} & 2 \\
I & I & F
\end{array}\right\} \delta\left(F^{\prime}, F^{\prime}\right) \delta\left(M_{F}, M_{F}^{\prime}\right) \delta\left(I, \frac{3}{2}\right) \\
& Q=(-)_{1}^{\prime}+\mathrm{I}+\mathrm{F}+1\left[\frac{\left(2 \mathrm{~F}_{1}+1\right)\left(2 \mathrm{~F}_{1}^{\prime}+1\right)(2 \mathrm{~J}+1)(2 \mathrm{~J}+2)(2 \mathrm{~J}+3) \mathrm{I}_{4}\left(\mathrm{I}_{4}+1\right)\left(2 \mathrm{I}_{4}+1\right)}{2 \mathrm{~J}(2 \mathrm{~J}-1)}\right]^{\frac{1}{2}} \times
\end{aligned}
$$

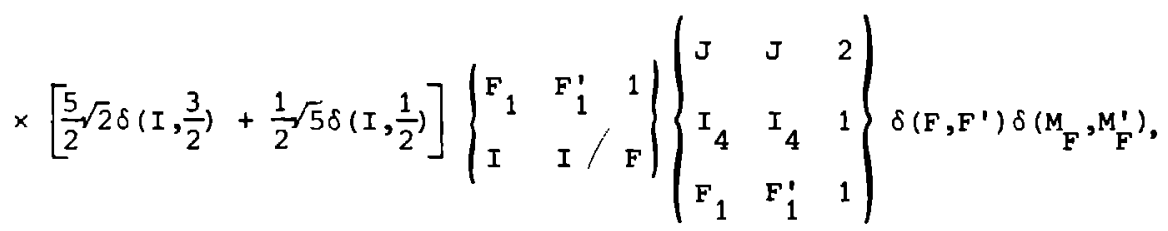

where $I=3 / 2$ for $A-1$ evels and $I=1 / 2$ for E-levels. The degeneracy of a hyperfine sublevel is $(2 \mathrm{~F}+1)$ and $2(2 \mathrm{~F}+1)$ for a rotational level 
of species $A$ and $E$, respectively. The mutual spin-spin interactions between the protons in the top vanish for E-levels. In deriving Eqs. (36) and (38), use is made of the fact that the matrix elements should be invariant under a cyclic permutation of the protons in the top.

B. THE HYPERFINE COUPLING CONSTANTS.

All coupling constants can be written in terms of $<J_{\gamma \pm}|| T^{(\nu)}|| J_{\gamma \pm}>$, where $T^{(\nu)}$ are spherical tensors of rank $\nu=0$, 1 or 2 (Eqs.(39-41)). These matrix elements can be evaluated with the aid of the Wigner-Eckart theorem :

$$
\begin{aligned}
& <J_{\gamma \pm}|| T^{(\nu)}|| J_{\gamma \pm}>=(-){ }^{J-M_{J}}\left(\begin{array}{ccc}
J & \nu & J \\
& & \\
-M_{J} & 0 & M_{J}
\end{array}\right)^{-1}\left\langle J_{Y \pm} M_{J}\left|T_{0}^{(\nu)}\right| J_{Y \pm} M_{J}\right\rangle \\
& =\left(\begin{array}{ccc}
J & \nu & J \\
-J & 0 & J
\end{array}\right)^{-1}<J_{\gamma \pm} J\left|T_{0}^{(\nu)}\right| J_{\gamma \pm} J>.
\end{aligned}
$$

The transformation of spherical tensor $T^{(\nu)}$ from the space-fixed to the molecular frame of reference, in which the tensor components are explicitly known, is given by:

$$
T_{q^{\prime}}^{(v)}=\sum_{q}(-)^{q} D_{q^{\prime}, q}^{(\nu)}\left(\psi, \theta, \phi_{1}\right) \underset{q}{q} \underset{q}{q}
$$

where $\underset{T}{q}(\nu)$ are the tensor components in the molecular frame, and $D_{q^{\prime}, q}^{(\nu)}\left(\psi, \theta, \phi_{1}\right)$ are the elements of the representation matrix of 3dimensional rotation. Hence:

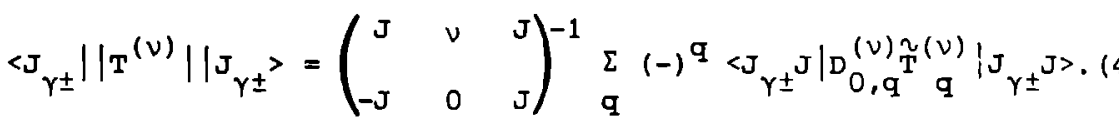

This expression can be evaluated for molecules which are of nearly 
symmetric-top type. In that case the rotational wavefunctions, which are serles expansions (Appendix), can be approximated by (Eq. (A16), Appendix):

$$
\left|J_{\gamma \pm J_{J}}\right\rangle=\left|J_{J^{\prime}} K \tau \pm\right\rangle=\frac{1}{\sqrt{2}}\left(u_{K \tau}^{J M} \pm u_{-K \tau}^{J M}\right)
$$

with $\mathrm{K}>0$ and $\left(\tau, \tau^{\prime}\right)=(1,1),(2,3)$ or $(3,2)$, depending on the value of $K$ ( $M$ stands for $M_{J}$ ). The only exception is the A-type rotational level with $K=0$, which we shall exclude for the present, since the results are the same. The approximation Eq. (45) is extremely good for the rotational wavefunctions of $\mathrm{CH}_{3} \mathrm{OH}$ with relatively low Jvalues (11). As pointed out in the Appendtx, the subscript $\gamma$ can be replaced by $K \tau$. The wavefunctions $u_{K \tau}^{J M}$ as given in Eq. (A6) can be rewritten in terms of the Euler angles $\psi, \theta$ and $\phi_{1}$ of the moleculefixed coordinate system defined in this section:

$$
\mathrm{u}_{\mathrm{KT}}^{\mathrm{JM}}=|\mathrm{JKM}\rangle\left|\tau_{\mathrm{K}}\right\rangle
$$

where |JKM> are the symmetric top eigenfunctions and:

$$
\left|\tau_{K}\right\rangle=\exp (-i K \alpha) p^{K \tau}(\alpha)
$$

where:

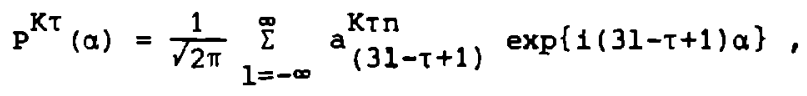

The coefficients $a_{s}^{K \tau n}$ are defined in the Appendix. The difference in the expression of $\left|\tau_{K}\right\rangle$ above with the expression (A6) originates from the different choice of the molecular frame $(x, y, z$ here and $a, b, c$ in the Append $1 x)$. With the wavefunctions defined in Eqs. (45) and (46), Eq. (44) becomes now: 
$\left\langle\mathrm{JK} \tau \pm \| \mathrm{T}^{(\nu)}|| \mathrm{JK} \tau \pm\right\rangle=\frac{1}{2}\left(\begin{array}{ccc}J & \nu & \mathrm{J} \\ -\mathrm{J} & 0 & \mathrm{~J}\end{array}\right)^{-1} \times$

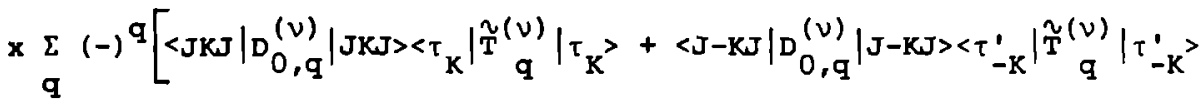

$\left.\pm\left\langle J K J\left|D_{0, q}^{(\nu)}\right| J-K J><\tau_{K}\left|\widetilde{T}_{q}^{(\nu)}\right| \tau_{-K^{\prime}}^{\prime}\right\rangle \pm\left\langle J-\left.K J\right|_{0, q} ^{(\nu)}\right| J K J>\left\langle\tau \tau_{-K}^{\prime}\left|\tilde{T}_{q}^{(\nu)}\right| \tau_{K}\right\rangle\right]$.

By integrating over $\psi, \theta$ and $\phi_{1}$ we get:

$\left\langle J K \tau \pm \| T^{(\nu)}|| J K \tau \pm\right\rangle=(-)^{J+K} \frac{1}{2}(2 J+1) x$

$x\left[\left(\begin{array}{ccc}J & \nu & J \\ -K & 0 & K\end{array}\right)\left\{\left\langle\tau_{K}\left|\widetilde{T}_{0}^{(\nu)}\right| \tau_{K}>+(-)^{\nu}<\tau_{-K}^{\prime}||_{0}^{(\nu)}\right| \tau_{-K}^{\prime}>\right\} \pm\right.$

$\pm\left(\begin{array}{ccc}J & 2 & J \\ -K & 2 & -K\end{array}\right)\left\{\left\langle\tau_{K}\left|\tilde{T}_{2}^{(2)}\right| \tau_{-K^{\prime}}^{\prime}\right\rangle+\left\langle\tau_{-K}^{\prime}\left|\tilde{T}_{-2}^{(2)}\right| \tau_{K}>\right\} \delta(K, 1) \delta(\nu, 2)\right]$.

Substitution of this equation into Eqs. (39), (40) and (41), and evaluation of the $3-y$ symbols yields:

$$
\begin{aligned}
C M_{J K \tau \pm}=\frac{1}{2 J(J+1)} & <\tau_{K}\left|\left\{J(J+1)-K^{2}\right\}\left(M_{X X X}+M_{Y Y}\right)+2 K^{2} M_{Z Z}\right| \tau_{K}> \\
& \pm \frac{1}{2} \operatorname{Re}<\tau_{K}\left|M_{2}^{(2)}\right| \tau_{-K}^{1}>\delta(K, 1),
\end{aligned}
$$

$\operatorname{Cd}_{J K \tau \pm}=\frac{-K}{J(J+1)} \operatorname{Re}<\tau_{K}\left|\frac{p d_{Z}+d_{Z} P}{2}\right| \tau_{K}>$

$S_{\mathrm{JK} \pm \pm}=\frac{\sqrt{6}}{3(\mathrm{~J}+1)(2 \mathrm{~J}+3)}\left\{3 \mathrm{~K}^{2}-\mathrm{J}(\mathrm{J}+1)\right\}\left\langle\tau_{\mathrm{K}}\left|\mathrm{D}_{0}^{(2)}\right| \tau_{\mathrm{K}}\right\rangle \pm$

$$
\pm \frac{J}{2 J+3} \operatorname{Re}<\tau_{K}\left|D_{2}^{(2)}\right| \tau_{-K}^{\prime}>\times \delta(K, 1) .
$$

For simplicity we have dropped from these formulae the characters, which label the nuclel. In deriving these equations, use has been made of the fact that: $\left|\tau_{-K^{\prime}}^{\prime}\right\rangle^{M}=\left|\tau_{K}\right\rangle,\left(\begin{array}{c}(2) \\ 0\end{array}\right)^{M}=\underset{0}{(2)} ;\left(M_{-2}^{(2)}\right)^{*}=$ 
$=M_{2}^{(2)} ;\left(D_{0}^{(2)}\right)^{x}=D_{0}^{(2)} ;\left(D_{-2}^{(2)}\right)^{x}=D_{2}^{(2)} ;\left(d_{z}\right)^{x}=d_{z}$ and $p^{x}=-p$.

Substitution of Eqs. (49), (50), and (51) 1nto Eqs. (37) and (38) gives the expressions for the hyperfine coupling constants. The terms off-diagonal in $\tau_{K}$ vanish only for the rotational levels of species E. It can be proved that each proton in the top has an equal contribution to the coupling constants. This is also evident because of the symmetry.

The tensor components $\left[D_{K L}\right\}_{q}^{(2)}$ for $K, L=1,2,3$ depend on the angle $\alpha$ as $\exp (-i q \alpha)$. So $\left\{\mathrm{D}_{\mathrm{KL}}\right\}_{0}^{(2)}$ is independent on $\alpha$ and its contribution to the spin-spin coupling constant $\mathrm{D}_{\mathrm{J} Y \pm}^{(1)}$ can be calculated from the geometry of the molecule. The contribution of $\left\{\mathrm{D}_{\mathrm{KL}}\right\}_{2}^{(2)}$, however, vanishes which can be shown by calculation. The value of the first spin-spin constant is then:

$$
D_{J K_{\tau} \pm}^{(1)}=\frac{3 \mu_{0} \mu_{N}^{2} g_{H}^{2}}{4 \pi}\left[\frac{3 K^{2}-J(J+1)}{(J+1)(2 J+3)}\right] r_{H H}^{-3},
$$

where $r_{H H}$ is the distance between two protons in the top. The second spin-spin coupling constant $D_{J Y( \pm)}^{(2)}$ can be determined numerically by calculating the relevant integrals over $\alpha$, if the geometry and the wavefunctions $\left|\tau_{\mathrm{K}}\right\rangle$ are known. This has been performed for some levels of the methanol molecule using a computer (11).

In the last section we have excluded for simplicity the levels with $k=0$. The coupling constants for these levels, however, can be calculated in an analogous way as for other $\mathrm{K}$-values. The results are also given by Eqs. (49), (50), (51), and (52) if we drop the \pm signs labeling the coupling constants. 
APPENDIX.

THE EIGENFUNCTIONS OF $\mathrm{H}_{0}$.

The Hamiltanian $\mathrm{H}_{0}$ is given in Eq. (28). The orientation of the molecule-fixed frame of reference $(x, y, z)$ relative to the spacefixed frame $(X, Y, Z)$ is described by the Euler angles $\psi, \theta$, and $\phi_{1}$. Using the convention of Edmonds (12) for the Euler angles we can write for the components of the angular momentum operators $\bar{j}$ and p in the $(x, y, z)$ frame:

$J_{x}=-i \hbar\left\{-\sin \phi_{1} \cot \theta\left(\frac{\partial}{\partial \phi_{1}}\right)_{\psi, \theta, \alpha}+\cos \phi_{1}\left(\frac{\partial}{\partial \theta}\right)_{\psi, \phi_{1}, \alpha}+\frac{\sin \phi}{\sin \theta}\left(\frac{\partial}{\partial \psi}\right)_{\phi_{1}, \theta, \alpha}\right\}$ $J_{y}=-i \hbar\left\{-\cos \phi_{1} \cot \theta\left(\frac{\partial}{\partial \phi_{1}}\right)_{\psi, \theta, \alpha}-\sin \phi_{1}\left(\frac{\partial}{\partial \theta}\right)_{\psi_{,} \phi_{1}, \alpha}+\frac{\cos \phi_{1}}{\sin \theta}\left(\frac{\partial}{\partial \psi}\right)_{\phi_{1}, \theta, \alpha}\right\}$ $J_{z}=-1 \hbar\left(\frac{\partial}{\partial \phi_{1}}\right)_{\psi, \theta, \alpha}$

$p=-i \hbar\left(\frac{\partial}{\partial \alpha}\right) \dot{\psi}, \theta, \phi_{1}$

These components satisfy the commutation relations:

$$
\begin{aligned}
& {\left[J_{i}, J_{j}\right]=-i \hbar J_{k}(i, j, k=x, y, z \text { in cyclic order })} \\
& {\left[J_{i}, p\right]=0}
\end{aligned}
$$

In order to reduce the coupling between $J_{z}$ and $p$ by a factor $\frac{D^{2}}{B C}$ the Nielson transformation is applied:

$\theta^{\prime}=\theta, \quad \psi^{\prime}=\psi, \quad \phi^{\prime}=\phi_{1}-\frac{C_{2}}{c} a, \quad a^{\prime}=\alpha, \quad$

( $\alpha$ is related to the Euler angles $\phi_{1}$ and $\phi_{2}$ of the two groups of the molecule as: $\left.\alpha=\phi_{1}-\phi_{2}\right)$. This transformation is equivalent to a 


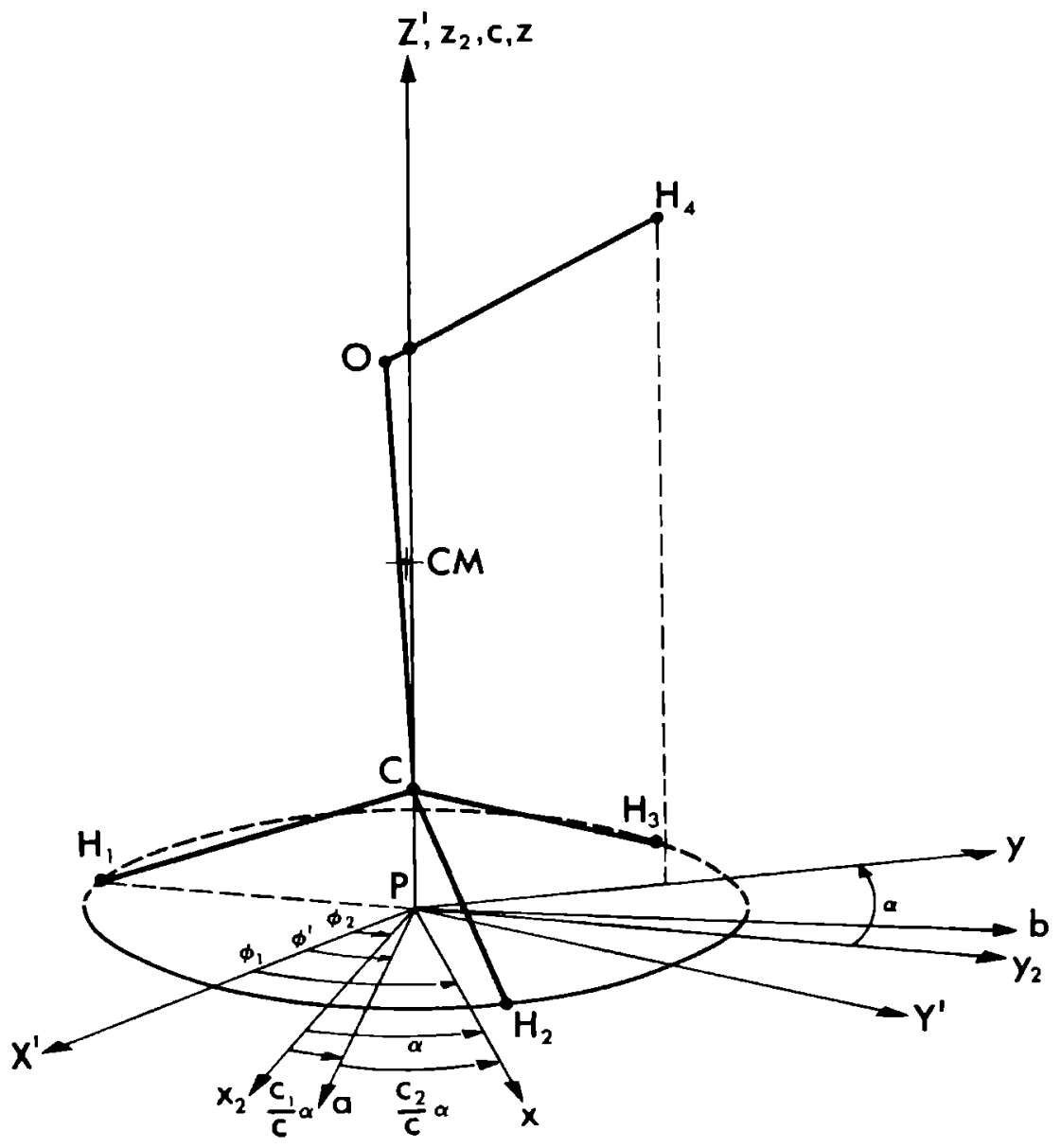

Fig.1. The methanol molecule with coordinate systems: $(x, y, z)$ fixed to the "frame", $\left(x_{2}, y_{2}, z_{2}\right)$ fixed to the "top", and $(a, b, c)$ fixed to the molecule. The coordinate system $\left(X^{\prime}, Y^{\prime}, Z^{\prime}\right)$ is the space fixed coordinate system $(X, Y, Z)$ after a rotation over the Euler angle $\psi$ about the z-axis, followed by a rotation over the Euler angle $\theta$ about the new Y'-axis. All coordinate systems have their origin in the center of mass (CM). For clarity they are drawn after a translation of the origin to $P$. 
rotation about the $z$-axis over an angle $\frac{C_{2}}{C} \alpha$, resulting in a coordinate system $(a, b, c)$ with Euler angles $\psi^{\prime}, \theta^{\prime}$, and $\phi^{\prime}(F 1 g, 1)$. This system is molecule-fixed, but not longer fixed to the frame (or to the top). The angular momentum operators $J_{a^{\prime}} J_{b^{\prime}} J_{c^{\prime}}$ and $P^{\prime}$ In the new frame are also given by Eq. (A1) if $\psi, \theta, \phi_{1}$ and $\alpha$ is replaced by $\psi^{\prime}, \theta^{\prime}, \phi^{\prime}$ and $\alpha^{\prime}$, respectively, and the same commutation relations hold. Both sets of angular momenta are related by the following expressions:

$$
\begin{aligned}
& J_{x}=\cos \left(\frac{C_{2}}{C} \alpha\right) J_{a}+\sin \left(\frac{C_{2}}{C} \alpha\right) J_{b}, \\
& J_{y}=-\sin \left(\frac{C_{2}}{C} \alpha\right) J_{a}+\cos \left(\frac{C_{2}}{C} \alpha\right) J_{b}, \\
& J_{z}=J_{C^{\prime}}, \\
& P=P^{\prime}-\frac{C_{2}}{C} J_{C} .
\end{aligned}
$$

By substituting Eqs.(A1)-(A4) Into Eq. (28) the latter equation transforms into:

$$
\mathrm{H}_{0}=\mathrm{H}_{I}+\mathrm{H}_{I I}+\mathrm{H}_{I I I}^{+}+\mathrm{H}_{I I I}^{-}+\mathrm{B}_{I V}^{+}+\mathrm{H}_{I V}^{-},
$$

w1th:

$$
\begin{aligned}
& B_{I}=\frac{(A+B) C_{1}-D^{2}}{4 A\left(B C_{1}-D^{2}\right)}\left(J^{2}-J_{C}^{2}\right)+\frac{1}{2 C} J_{C}^{2}+\frac{C}{2 C_{1} C_{2}} p^{\prime 2}+V(\alpha) \text {, } \\
& \mathrm{H}_{I I}=\frac{\mathrm{D}^{2}}{2 \mathrm{C}_{1}\left(B \mathrm{C}_{1}-\mathrm{D}^{2}\right)}\left[\frac{\mathrm{C}_{1}^{2}}{\mathrm{C}^{2}} \mathrm{~J}_{\mathrm{C}}^{2}+\frac{2 C_{1}}{\mathrm{C}} \mathrm{p}^{\prime} \mathrm{J}_{\mathrm{C}}+\mathrm{P}^{\prime 2}\right] \text {, } \\
& \mathrm{B}_{\mathrm{III}}^{ \pm}=\frac{\overline{ \pm} 1 \mathrm{D}}{2\left(B C_{1}-D^{2}\right)} \exp \left( \pm i \frac{C_{2}}{C} a\right) J_{ \pm}\left( \pm \frac{1}{2} \hbar+P^{\prime}+\frac{C_{1}}{C} J_{c}\right), \\
& H_{I V}^{ \pm}=\frac{(A-B) C_{1}+D^{2}}{8 A\left(B C_{1}-D^{2}\right)} \exp \left( \pm 21 \frac{C_{2}}{C} \alpha\right) J_{ \pm}^{2} \text {. }
\end{aligned}
$$


with:

$$
J_{ \pm}=-\left(J_{a} \mp i J_{b}\right)
$$

The first term $\left(\mathrm{H}_{I}\right)$ of the Hamlltonian $\mathrm{H}_{0}$ is a Hamiltonian of a symmetric top molecule with internal rotation. The terms $\mathrm{H}_{\text {II' }} \mathrm{H}_{\text {III }}^{ \pm}$ and $\mathrm{H}_{\mathrm{IV}}^{ \pm}$are due to the asymmetry of the molecule. They can be considered as small pertubations on $\mathrm{H}_{\mathrm{I}^{\prime}}$ if the molecule is nearly a symmetric top as in the case of $\mathrm{CH}_{3} \mathrm{OH}$. The eigenfunctions of $\mathrm{H}_{\mathrm{I}}$ have been calculated by Koehler and Dennison (13), and Verhoeven (14). Their result is:

$$
\left.u_{K \tau \Omega}^{J M}=|J K M>| \tau_{K \Omega}\right\rangle,
$$

where $\mid J K M>$ are the normal symmetric top wavefunctions and:

$$
\mid \tau_{K \Omega}>=\exp \left(-i K \frac{C_{1}}{C} \alpha\right) P^{K \tau n}(\alpha)
$$

with:

$$
\mathbf{P}^{\mathrm{K} \tau \mathrm{n}}(\alpha)=\frac{1}{\sqrt{2 \pi}} \sum_{1=-\infty}^{\infty} \mathrm{a}_{(31-\tau+1)}^{\mathrm{K} \tau \mathrm{n}} \exp \{\mathrm{i}(3 l-\tau+1) \alpha\} ; \tau=1,2,3,
$$

are the solutions of the Mathieu differential equations. The coefficients $a_{s}^{K \tau_{n}}$ in the Fourrier-expansion are tabulated for methanol in Refs. (15) and (16). In the following we shall restrict ourselves to $n=0$, and drop $n$ from $u_{K \tau_{n}}^{J M}$ and $\left|\tau_{K n}\right\rangle$.

For the calculation of the matrix elements of $\mathrm{H}$ on this basis of $u_{K \tau}^{J M}$ we consider symmetry properties of these functions under the operations of the symmetry group of the Hamiltonian, in order to determine the structure of the energy matrix.

The group operations as described in Eq. (30) are in terms of the Euler angles $\left(\psi^{\prime}, \theta^{\prime}, \phi^{\prime}\right)$ and $\alpha^{\prime}$ given by: 


$$
\begin{aligned}
& \mathrm{E}: \psi^{\prime} \rightarrow \psi^{\prime} ; \theta^{\prime} \rightarrow \theta^{\prime} \quad ; \phi^{\prime} \rightarrow \phi^{\prime} \quad ; \alpha^{\prime} \rightarrow \alpha^{\prime} \\
& \mathrm{C}_{3}: \psi^{\prime} \rightarrow \psi^{\prime} \quad ; \theta^{\prime} \rightarrow \theta^{\prime} \quad ; \phi^{\prime} \rightarrow \phi^{\prime}-\frac{2 \pi}{3} \frac{\mathrm{C}_{1}}{\mathrm{C}} ; \alpha^{\prime} \rightarrow \alpha^{\prime}+\frac{2 \pi}{3} \\
& \mathrm{C}_{2 \mathrm{x}}: \psi^{\prime} \rightarrow \psi^{\prime}+\pi ; \theta^{\prime} \rightarrow \pi-\theta^{\prime} ; \phi^{\prime} \rightarrow 2 \pi-\phi^{\prime} ; \alpha^{\prime} \rightarrow-\alpha^{\prime}
\end{aligned}
$$

A set of functions $u_{K T}^{J M}$ with the same $J, M$ and absolute value of $K$ ( 1 ix if $K \neq 0$ and three if $\mathrm{K}=0$ ) form a basis for a reducible representation of the group. This can be proved by using the relations $a_{s}^{K 1}=a_{-s}^{-K 1}$ and $a_{s}^{K 2}=a_{-s}^{-K 3}$ of the coefficients in the expansion of $\left|\tau_{K}\right\rangle$. The result of the decomposition of the representation into its constituent irreducible representations, together with the correct basis functions are given in Table III.

All functions $\Psi$ as given in Table III transform under the $C_{3}$ groupoperation as $C_{3} \Psi=\exp \left(\frac{2 \pi}{3} \sigma\right.$ i) $\Psi$. The $A_{1}$ functions are symmetric under the $C_{2 x}$ operation and the $A_{2}$ functions are antisymetric. $A$ set of E type functions $\left(\Psi_{1}, \Psi_{2}\right)$ transforms under the $C_{2 x}$ operation as: $c_{2 x} \Psi_{1}=(-)^{J_{\Psi}}$ and $C_{2 x} \Psi_{2}=(-)^{J_{\Psi}}$. Since the Hamiltonian $H_{0}$ is clearly of species $A_{1}$, we can use the results obtained by the wigner Eckart theorem (9). The matrix of the Hamiltonian reduces to four submatrices, the first one with basis functions of species $A_{1}(\sigma=0)$, the second one with basis functions of species $A_{2}(\sigma=0)$ and the last two, which are identical, with basts functions of species $E$ $(\sigma=1$ and $\sigma=-1$, respectively) The elgenfunctions of $\mathrm{H}_{0}$ are*) :

*) These elgenfunctions are in perfect agreement with those of Verhoeven (14) but not with those given by Ivash et al (15). Although both authors calculate them in the same manner (not using group theory) the difference may be a question of phase convention. 
Table III. The basis functions $\Psi$ of the irreducible representations (column 1 ) of the group $c_{3 v}$; $=0,1$, $2, \ldots$ : o (last column) determines the symmetry of $\Psi$ under the group operation $C_{3}\left(C_{3} \Psi=\exp \left(\frac{2 \pi}{3} \sigma 1\right) \Psi\right)$.

\begin{tabular}{|c|c|c|c|c|c|}
\hline \multirow{2}{*}{ REPRESENTATION } & \multicolumn{4}{|c|}{ BASIS FUNCTIONS $\Psi$} & \multirow{2}{*}{$\sigma$} \\
\hline & $\mathrm{K}=0$ & $K=3 n \quad(n \neq 0)$ & $K=3 n+1$ & $k=3 n+2$ & \\
\hline$A_{1}$ & \multirow{2}{*}{$\mathrm{u}_{01}^{\mathrm{JM}}{ }^{\mathrm{a}}$} & $\frac{1}{\sqrt{2}}\left\{\mathrm{u}_{\mathrm{K} 1}^{\mathrm{JM}}+(-)^{\mathrm{J}} \mathrm{u}_{-\mathrm{K} 1}^{\mathrm{JM}}\right\}$ & $\frac{1}{\sqrt{2}}\left\{u_{\mathrm{K} 3}^{\mathrm{JM}}+(-) \mathrm{u}_{-\mathrm{K} 2}^{\mathrm{JM}}\right\}$ & $\frac{1}{\sqrt{2}}\left\{\mathrm{u}_{\mathrm{K} 2}^{\mathrm{JM}}+(-)^{\mathrm{J}} \mathrm{u}_{-\mathrm{K} 3}^{\mathrm{JM}}\right\}$ & 0 \\
\hline $\mathrm{A}_{2}$ & & $\frac{1}{\sqrt{2}}\left\{u_{K 1}^{J M}-(-)^{J} u_{-K 1}^{J M}\right\}$ & $\frac{1}{\sqrt{2}}\left\{u_{K 3}^{J M}-(-)^{J} u_{-K 2}^{J M}\right\}$ & $\frac{1}{\sqrt{2}}\left\{u_{K 2}^{J M}-(-)^{J} u_{-K 3}^{J M}\right\}$ & 0 \\
\hline $\mathbf{E}$ & $\begin{array}{l}u_{03}^{J M} \\
u_{02}^{J M}\end{array}$ & $\begin{array}{c}\mathrm{u}_{\mathrm{K} 3}^{\mathrm{JM}} \\
\mathrm{u}_{-\mathrm{K} 2}^{\mathrm{JM}}\end{array}$ & $\begin{array}{c}\mathrm{u}_{\mathrm{K} 2}^{\mathrm{JM}} \\
\mathrm{u}_{-\mathrm{K} 3}^{\mathrm{JM}}\end{array}$ & $\begin{array}{c}\mathrm{u}_{\mathrm{K} 1}^{\mathrm{JM}} \\
\mathrm{u}_{-\mathrm{K} 1}^{\mathrm{JM}}\end{array}$ & $\begin{array}{r}1 \\
-1\end{array}$ \\
\hline $\mathbf{E}$ & & $\begin{array}{c}\mathrm{u}_{-\mathrm{K} 3}^{\mathrm{JM}} \\
\mathrm{u}_{\mathrm{K} 2}^{\mathrm{JM}}\end{array}$ & $\begin{array}{c}\mathrm{u}_{-\mathrm{K} 1}^{\mathrm{JM}} \\
\mathrm{u}_{\mathrm{K} 1}^{\mathrm{JM}}\end{array}$ & $\begin{array}{c}\mathrm{u}_{-\mathrm{K} 2}^{\mathrm{JM}} \\
\mathrm{u}_{\mathrm{K} 3}^{\mathrm{JM}}\end{array}$ & $\begin{array}{r}1 \\
-1\end{array}$ \\
\hline
\end{tabular}

a $U_{01}^{J M}$ is of species $A_{1}$ or $A_{2}$ for $J$ even or odd. 
$\left.\begin{array}{r}\text { (1) Species } A_{1}(J \text { even) } \\ \quad \text { or } A_{2} \text { (J odd ) }\end{array}\right\} \mid J_{\gamma+} M_{J}>=a_{0} u_{01}^{J M}+\underset{K>0}{\sum} \frac{a_{K}}{\sqrt{2}}\left(u_{K \tau}^{J M}+u_{-K T}^{J M},\right)$

$\left.\begin{array}{r}\text { (ii) Species } A_{2}(J \text { even) } \\ \text { or } A_{1}(J \text { odd })\end{array}\right\} \mid J_{Y-} M_{J}>=\sum_{K>0} \frac{b_{K}}{\sqrt{2}}\left(u_{K \tau}^{J M}-u_{-K \tau}^{J M},\right)$,

where:

$$
\begin{aligned}
& \tau=1, \tau^{\prime}=1 \text { if } k=3 n \text { with } n=1,2,3, \ldots \\
& \tau=3, \tau^{\prime}=2 \text { if } k=3 n+1 \text { with } n=0,1,2, \ldots \\
& \tau=2, \tau^{\prime}=3 \text { if } k=3 n+2 \text { with } n=0,1,2, \ldots
\end{aligned}
$$

These wavefunctions belong to the energy levels denoted by ( $\mathrm{J}_{\gamma+}$ ) or $\left(J_{\gamma-}\right)$, where $\gamma$ labels the $A_{1}$ or $A_{2}$-levels with the same $J$.

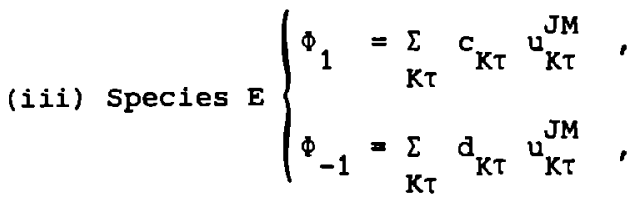

where the sums run over all values of $K T$ with $\sigma=1$ or $\sigma=-1$ for $\Phi_{1}$ or $\Phi_{-1}$, respectively. The coefficients $c_{K \tau}$ and $d_{K^{\prime} \tau^{\prime}}$ are equal if the corresponding wavefunctions $u_{K \tau}^{J M}$ and $u_{K^{\prime} \tau}^{J M}$, form a basis for the E-representation (see Table III). Evexy linear combination of $\Phi_{1}$ and $\Phi_{-1}$ is also an eigenfunction of the Hamiltonian and forms together with another independent linear combination of $\Phi_{1}$ and $\Phi_{-1}$ a basis for the E-representation. In analogy with the A-levels, we choose for a particular E-level as eigenfunctions:

$$
\begin{aligned}
& \left|J_{\gamma \pm}\right\rangle=\frac{1}{\sqrt{2}}\left(\Phi_{1} \pm \Phi_{-1}\right) \text { or: } \\
& \text { E-level }\left\{\begin{array}{l}
\mid J_{\gamma^{+}} M_{J^{\prime}}>=\sum_{K} c_{K \tau} \frac{1}{\sqrt{2}}\left(u_{K \tau}^{J M}+u_{-K \tau}^{J M},\right), \\
J_{\gamma^{-}} M_{J^{\prime}}>=\sum_{K} c_{K \tau} \frac{1}{\sqrt{2}}\left(u_{K \tau}^{J M}-u_{-K \tau}^{J M}\right),
\end{array},\right.
\end{aligned}
$$


with:

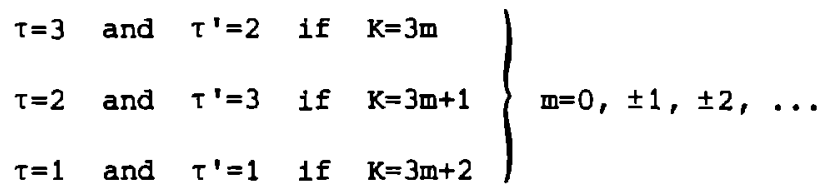

The corresponding levels are denoted by $\left(J_{\gamma}\right)$, where $\gamma$ labels the levels with the same J. Both choices (Eqs. (A11) and (A12)) of the E-levels are, of course, equivalent. The first one (Eq.(A11)) 1s the most common in litterature; all functions have a definite parity for the $c_{3}$ operation $\left(\exp \left(\frac{2 \pi}{3} \sigma i\right)\right)$. We prefer the latter one (Eq. (A12)), because the calculation of the hyperfine structure is easier in that case; both the $A$ and $E$ eigenfunctions have a definite parity for the $\mathrm{C}_{2 \mathrm{x}}$ operation:

$$
C_{2 x}\left|J_{Y \pm} M_{J}\right\rangle= \pm(-)^{J}\left|J_{Y \pm} M_{J}\right\rangle
$$

The coefficients $a_{K \tau}, b_{K \tau}$ and $c_{K \tau}$ of the eigenfunctions can be calculated by diagonalizing the Hamiltonian matrix. The matrix elements are easily derived from Eq. (A5). They are given in the following equations, using the shorthand notation: $H_{J^{\prime} K^{\prime} \tau^{\prime}}^{J K}=\left\langle u_{K \tau}^{J M}\left|H_{0}\right| u_{K^{\prime} \tau^{\prime}}^{J M}\right\rangle$

$$
\begin{gathered}
H_{J K \tau}^{J K \tau}=\left(H_{I}\right)_{J K \tau}^{J K \tau}+\left(H_{I I}\right)_{J K \tau}^{J K \tau}=\hbar^{2} \frac{(A+B) C_{1}-D^{2}}{4 A\left(B C_{1}-D^{2}\right)}\left(J^{2}+J-K\right)+\frac{\hbar^{2}}{2 C} K^{2}+E^{K \tau}+ \\
+\hbar^{2} \frac{D^{2}}{2 C_{1}\left(B C_{1}-D^{2}\right)}\left[\frac{C_{1}^{2}}{C^{2}} K^{2}-\frac{C_{1}}{\pi C} 1 K \int_{0}^{2 \pi}\left\{P^{K \tau}(\alpha)\right\}^{M} \frac{d}{d \alpha} P^{K \tau}(\alpha) d \alpha-\right. \\
\left.-\frac{1}{2 \pi} \int_{0}^{2 \pi}\left\{P^{K \tau}(\alpha)\right\}^{M} \frac{d^{2}}{d \alpha^{2}} P^{K \tau}(\alpha) d \alpha\right],
\end{gathered}
$$

$H_{J K \pm 1 \tau}^{J K \tau},=\left(H_{I I I}^{\mp}\right)_{J K \pm 1 \tau^{\prime}}^{J K \tau}=\hbar^{2} \frac{1 D}{2\left(B C_{1}-D^{2}\right)}\{(J \mp K)(J \pm K+1)\}^{\frac{1}{2}} \times$ 
$\times \frac{1}{2 \pi} \int_{0}^{2 \pi}\left[\mp 1 e^{\mp 1 \alpha}\left\{p^{K \tau}(\alpha)\right\}^{M} \frac{d}{d \alpha} p^{K \pm 1 \tau^{\prime}}(\alpha)-\frac{1}{2} e^{\mp 1 \alpha}\left\{p^{K \tau}(\alpha)\right\}^{M} p^{K \pm 1 \tau^{\prime}}(\alpha)\right] d \alpha$

$$
\begin{aligned}
& H_{J K \pm 2 \tau}^{J K \tau}=\left(B_{I V}^{\mp}\right)_{J K \pm 2 \tau}^{J K \tau},=\hbar^{2} \frac{(A-B) C_{1}+D^{2}}{8 A\left(B C_{1}-D^{2}\right)}\{(J \mp K)(J \mp K-1)(J \pm K+1)(J \pm K+2)\}^{\frac{1}{2}} \\
& \quad \times \frac{1}{2 \pi} \int_{0}^{2 \pi}\left\{P^{K \tau}(\alpha)\right\}^{3 t} e^{\mp 21 \alpha} P^{K+2 \tau}(\alpha) d \alpha
\end{aligned}
$$

All other matrix elements are zero. The differences between the equations above and those of Ref. (17) are merely introduced by a different cholce of the Euler angles.

For slightly asymmetric top molecules the off-diagonal matrix elements are small. This implies that one term in the expansion of the eigenfunctions (Eqs.(A9), (A10), (A11), and (A12)) w1ll be dominant. This gives a possibility to specify in a unique way the label $\gamma$ of the energy levels and wavefunctions as: $\gamma=K \tau$ with $K>0$ if $\left(u_{K \tau}^{J M} \pm u_{-K \tau}^{J M},\right)$ is the dominant term. So levels of species $A$ are denoted by $\left(J K \tau \pm\right.$ ) with elgenfunctions $\mid J M_{J} K_{\tau \pm>}$ and the levels of species $\mathrm{E}$ are denoted by (JKT) with eigenfunctions $\left|\mathrm{JM}_{\mathrm{J}} \mathrm{K} \tau+\right\rangle$ and $\mid \mathrm{JM}_{\mathrm{J}} \mathrm{K \tau} \boldsymbol{} \rightarrow$

Molecules such as methanol are in very good approximation symmetric top molecules. In this case the wavefunctions both of $A$ and E-type levels can be approximated by the dominant term only (11):

$$
\left|\mathrm{JM}_{\mathrm{J}} \mathrm{K \tau} \pm\right\rangle=\frac{1}{\sqrt{2}}\left(\mathrm{u}_{\mathrm{K} \tau}^{\mathrm{JM}} \pm \mathrm{u}_{-K \tau}^{\mathrm{JM}},\right)
$$

with $K>0$ and $\left(\tau, \tau^{\prime}\right)=(1,1),(2,3)$ or $(3,2)$ depending on the value of $K$, except for the A-type levels with $K=0$, whose eigenfunctions are approximated by: 


$$
\left|J M_{J} 01\right\rangle=u_{01}^{J M} .
$$

These approximations are extremely good for the purpose of calculating the hyperfine coupling constants (of course not for the calculation of the rotational energies).

REFERENCES.

(1) G.R. Gunther-Mohr, C.H. Townes, and J.A. van Vleck, Phys.Rev. 94, 1191 (1954).

(2) A. Dymanus, Monograph No 1, Atomic and Molecular Research Group, Katholieke Universiteit, Nijmegen, (1966) .

(3) J.A.Th. Verhoeven, Thesis 1969, Katholieke Universiteit, Nijmegen.

(4) P. Thaddeus, I. Krisher, and J. Loubser, J.Chem. Phys. 40, 257 (1964).

(5) K.T. Hecht and D.M. Dennison, J.Chem.Phys. 26, 31 (1957).

(6) J.E.M. Heuvel, Quarterly Report No 23, Atomic and Molecular Research Group, Katholleke Unlversiteit, N1jmegen, (1969).

(7) C.C. Lin and J.D. Swalen, Rev.Mod.Phys. 31, 841 (1959).

(8) H.C. Longuet-Higgins, Mol.Phys, 6, 445 (1962).

(9) B.R. Judd, "Operator Techniques in Atomic Spectroscopy", McGraw-Hill Book Company, Inc., New York (1962).

(10) A. Yutsis, I. Levinson, and V. Vanagas, "Theory of Angular Momentum", Israel Program for Scientific Translations, Jerusalem (1962).

(11) J.E.M. Heuvel, Quarterly Report No 25, Atomic and Molecular Research Group, Katholieke Universiteit, Nijmegen, (1969). 
(12) A.R. Edmonds, "Angular Momentum in Quantum Mechanics", Princeton University Press, Princeton, New Jersey (1960).

(13) J.S. Koehler and D.M. Dennison, Phys.Rev, 37, 1006 (1940).

(14) J.A.Th. Verhoeven, Monograph No 2, Atomic and Molecular Research Group, Katholieke Universiteit, NiJmegen, (1966).

(15) E.V. Ivash and D.M. Dennison, J.Chem.Phys. 21, 1804 (1953).

(16) D.G. Burkhard and D.M. Dennison, J.Mol.Spectrosc. 3 , 299 (1959) .

(17) D.G. Burkhard and D.M. Dennison, Phys.Rev. 84, 408 (1951). 
MEASUREMENTS AND RESULTS ON $\mathrm{CH}_{3} \mathrm{OH}$

I. INTRODUCTION.

We have performed accurate measurements on the hyperfine structure of the following transitions of methanol $\left(\mathrm{CH}_{3} \mathrm{OH}\right):(\mathrm{JK} \tau)=$ $(0,0,1) \rightarrow(1,0,1)$ at about $48 \mathrm{GHz} ;(\mathrm{J}, 1,3-) \rightarrow(\mathrm{J}, 1,3+)$ for $\mathrm{J}=2$, 3 and 6 at about 2.5, 5, and $17 \mathrm{GHz}$, respectıvely. The last three transitions will be called the $J=2,3$ and 6 transitions in the following.

Except for the $J=0$ level all other energy levels of methanol involved in the transitions are split because of the hyperfine interactions discussed in Chap. 2. The number of sublevels is six for $J=1$ and elght for all $J>1$. Hence, the spectrum of the $J=0 \rightarrow 1$ transition consists of six hyperfine components, while the spectrum of the $J=2,3$ and 6 will contain a large number of them. Explicit calculation of the relative intensities shows that eight of these components will be by far dominant.

The hyperfine interactions are the nuclear spin-spin and spinrotation interactions, as described in the previous chapter. The strength of the spin-spin interactions can be calculated and is of the order of $10 \mathrm{kHz}$. The strength of the spin-rotation interactions cannot be calculated in advance, but is estimated to be of the same order of magnitude as in the rigid rotor molecules. The quadrupole hyperfine interactions are absent in normal methanol because ${ }^{12} \mathrm{CH}_{3} \mathrm{OH}$ contains only nuclel with spin 0 or $\frac{1}{2}$. Hence, the 
hyperfine splitting of the rotational transitions was expected to be of the order of $10 \mathrm{kHz}$.

II. SPECTROMETERS AND EXPERIMENTAL METHOD.

The $\mathrm{J}=2,3$ and 6 transitions were Investigated with the beam-maser spectrometer (BMS FIg.1) described by Bluyssen (1). The source of the molecular beam was a crinckly foil effuser, $1 \mathrm{~cm}$ in diameter, contalning about 4000 channels. The source pressure was a few Torr's resulting in a flux of $10^{19} \mathrm{~mol} / \mathrm{sec}$. The state selector was an electrostatic octupole, constructed of highly polished cylindrical stainless steel rods, $28 \mathrm{~cm}$ long. The positively and negatively charged rods are separately mounted on sheaves of perspex

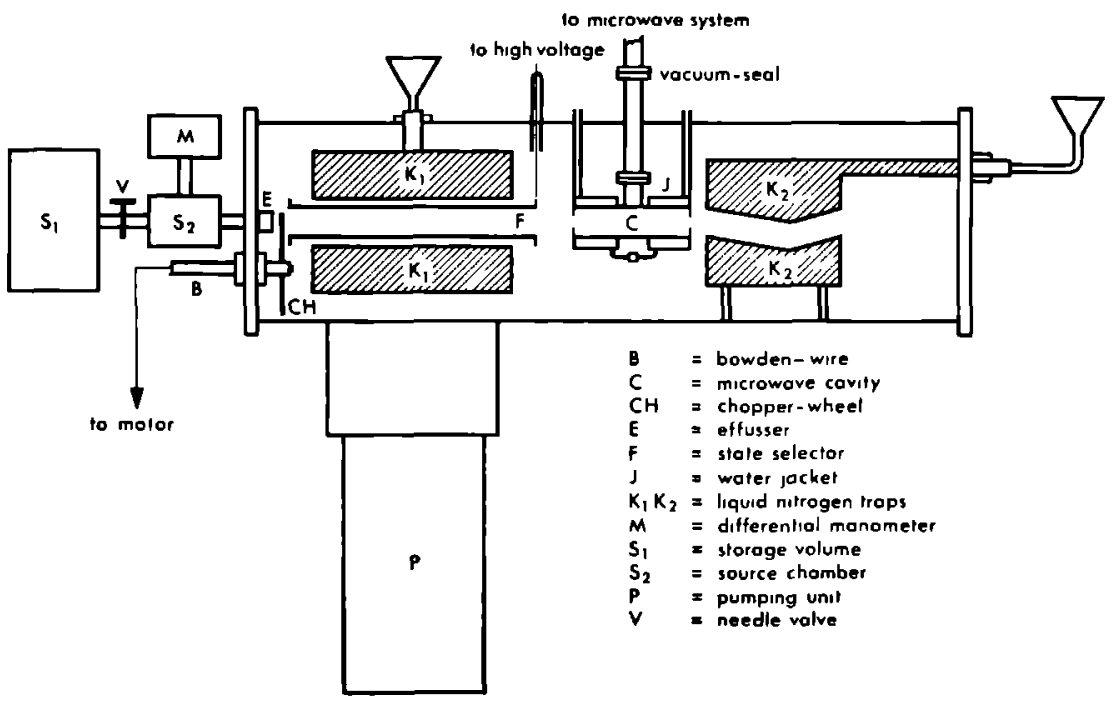

F1g.1. Schematic diagram of the beam-maser spectrometer (from: H. Bluyssen (1)). 
providing good electrical 1solation. The applied voltage was \pm 35 $\mathrm{kV}$ ylelding a maximum field of about $140 \mathrm{kV} / \mathrm{cm}$ between the neighbouring rods. The state selector was mounted inside a liquid nitrogen trap which trapped molecules deflected out of the beam. The experiments have been performed with cylindrical cavities, oscillating in the $T M_{010}$ mode. The length of the cavities was 28,100 , and $30 \mathrm{~cm}$ for the $\mathrm{J}=2,3$, and 6 transitions, respectively. The corresponding theoretical half-width due to the Heisenberg broadening is about $1.0,0.3$, and $0.9 \mathrm{kHz}$. The half-width could not be verified experimentally because none of the spectra contains a single line. The power emitted by the molecular beam $\left(10^{-10}-10^{-12}\right.$ W) was detected by a superheterodyne system with phase sensitive demodulation. Modulation is achieved by mechanlcal chopping of the beam at $120 \mathrm{~Hz}$. The detection scheme is shown in Fig. 2. Separate signal (SO) and local oscillator (LO) klystrons are employed with an intermediate frequency of $30 \mathrm{MHz}$. An automatic frequency control system using Schomandl FDS30 syncriminator phase-locks the LOklystron at $30 \mathrm{MHz}$ off the so-frequency. The so-klystron is coupled in the same way to a combined Schomandl-Rohde \& Schwarz frequency system. The latter system consists of the Schomandl ND30M variable oscillator ( $300 \mathrm{~Hz}-31 \mathrm{MHz}$ ) and the Rohde \& Schwarz XUC frequency synthesizer (470-1000 MHz) both driven by a $10 \mathrm{MHz}$ signal from the Rohde \& Schwarz XSU oscillator. This oscillator is coupled to a Varian R20 rubidium frequency standard ( $5 \mathrm{MHz}$ ). At the crystal M higher harmonics of the frequency of the synthesizer are generated and mixed with the so-frequency. The resulting $30 \mathrm{MHz}$ beat frequency is used for the stabilization of the so-klystron by a FDS30 


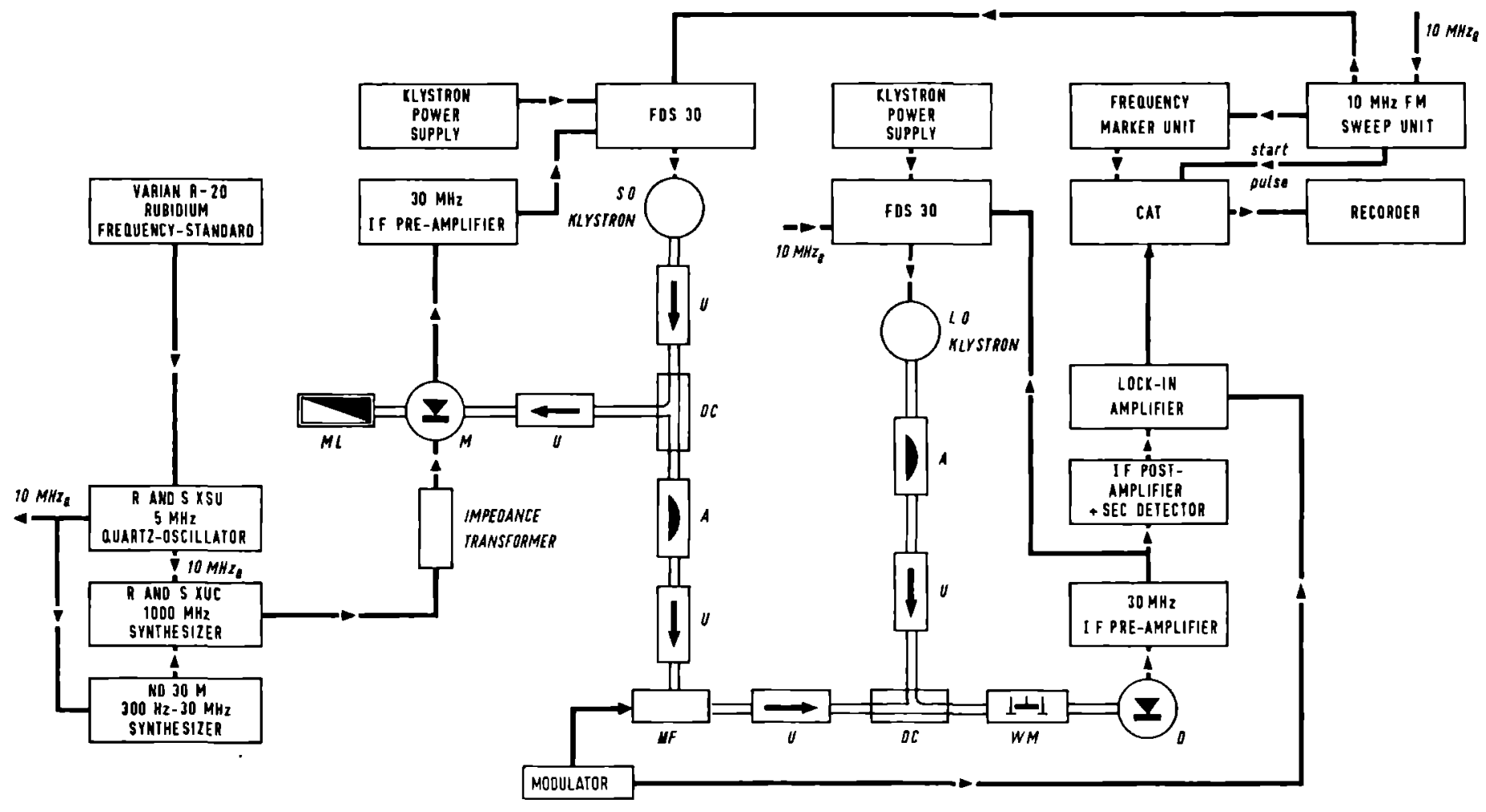

Fig.2. Superheterodyne detection scheme; $A=$ attenuator; $D C=$ directional coupler; $U$ = ferrite isolator; $M I=$ matched load; $D=$ mixer crystal; $M=$ multiplier crystal; $W M$ = wave meter; $M F=$ microwave $f i e l d:$ cavity in the BMS or parallel plate cell in the BAS; Modulator = beam chopper in the BMS or square wave Stark generator in the BAS. 
syncriminator.

For all investigated transitions the signal to nolse ratio at the output of the lock-in amplifier was insufficient for good results. In order to increase the signal to nolse ratio, time averaging techniques were employed with a computer of average transients (CAT, Technical Measurements Corporation). Exactly the same frequency region of the spectrum (typically $50-100 \mathrm{kHz}$ ) was scanned in a period of 20 seconds and added in the memory of the CAT (400 channels). The scanning was accomplished by sweeping the $10 \mathrm{MHz}$ reference signal of the FDS30 syncriminator of the so-klystron with a signal generated in a $10 \mathrm{MHz}$ sweep unit. A typical number of scans was 100 - 200. The RC-time of the lock-in amplifier was set at 0.3 or 1 second, depending on the frequency region. With the CAT the signal to noise ratio increases as the square root of the number of scans. A detailed description of the detection system is found in Ref. (ㅁ).

The reflex klystrons used for the transitions at $2.5,5$ and 17 GHz were Sanders no 6455, Varian $\mathrm{x} 26 \mathrm{E}$ and Varian $\mathrm{x} 12$, respectively. The signal to noise ratio of the measured spectra of the $J=$ 3 and 6 transitions was about 50, of the $J=2$ transition about 25 . The latter transition was investigated with a coaxial microwave system, the other ones with waveguide systems.

The $J=0 \rightarrow 1$ transition at $48 \mathrm{GHz}$ was investigated with the beam-absorbtion spectrometer (BAS) developed by Huiszoon $(\underline{3}, \underline{4})$. As a detailed description of the spectrometer can be found in the references mentioned above, only a general outline is given below (Fig.3). The absorption cell consists of two electrically isolated 


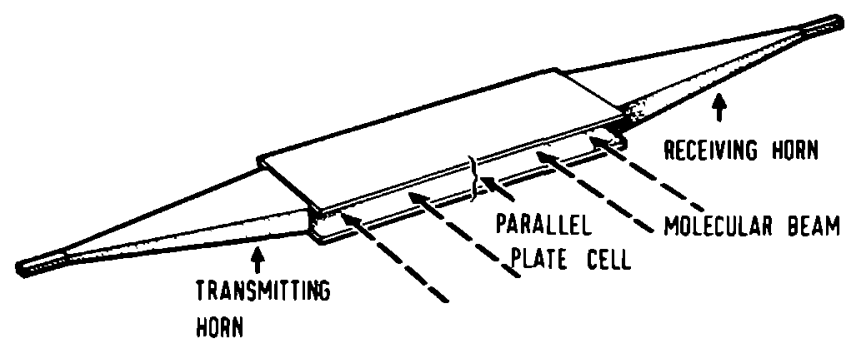

Fig.3. The absorption cell with horns. The direction of the molecular beam is indicated by arrows (from: C.Huiszoon ( 3 )).

flat copper plates, $40 \mathrm{~cm}$ long, $10 \mathrm{~cm}$ wide, separated by a distance of $1 \mathrm{~cm}$. The microwave radiation is sent between the plates by a transmitting horn and received by an identfcal horn. The aperture of the horns is $10 \times 90 \mathrm{~mm}$ and the length is $300 \mathrm{~mm}$. The horns are made of copper plates, simply screwed together. Between the horns and the cell, two cylindrical lenses are mounted, providing flat wavefronts of the microwave field propagating through the cell. The lenses are made of Rexolite 1422 (American Enkalon Division, USA). The propagation mode is essentially TEM with the E-vector perpendicular to the plates. The source of the molecular beam is a crinckly foil effuser $350 \mathrm{~mm}$ long and $4 \mathrm{~mm}$ wide, containing about 16000 channels. Between the effuser and the parallel plate cell a diaphragm at liquid nitrogen temperature serves to trap molecules not travelling in the direction perpendicular to the direction of the propagation of the microwave radiation. In the diaphragm are mounted parallel copper screens, $50 \mathrm{~mm}$ long, at a distance of $2 \mathrm{~mm}$ from each other. These screens form about 150 channels through which the molecules have to travel before entering the parallel 
plate region. The divergence of the beam in the direction of propagation of the radiation is estimated to be $1 / 25$ radians. Its contxibution to the Doppler broadening results in a half-width $\left(\Delta \nu_{D}\right)$ of $2.1 \mathrm{kHz}$ for $\mathrm{CH}_{3} \mathrm{OH}$ at $48 \mathrm{GHz}$. The half-width $\left(\Delta \nu_{\mathrm{H}}\right)$ due to the Heisenberg broadening is $1.7 \mathrm{kHz}$ at room temperature. The experimental half-width was about $3.0 \mathrm{kHz}$. The detection system is essentially the same as that described above for the beam-maser spectrometer. The microwave cavity in the BMS is replaced by the parallel cell in the BAS, (the cavity has one single hole for the in- and output of the microwave radiation, while the parallel cell is connected to separate transmitting and receiving horn, but this is not an essential difference). The modulation of the signal is achieved by applying a square-wave stark voltage (500 V, $1072 \mathrm{~Hz}$ ) between the plates of the cell. As signal and local oscillators we used two OKI reflex klystrons type $45 \mathrm{~V} 10$.

The measurements of the $J=1 \rightarrow 0$ transitions by the BAS were laborous, because the permeability of the channels in the diaphragm decreases quickly during operation due to the sticking of methanol molecules on the inner surfaces of the channels. The effective measuring time was about 10-15 minutes a day. Several days had to be spent to obtain one spectrum. We took special care to keep the frequency sweep region of the spectrum constant during the accumulation time of the CAT. The signal to noise ratio with the CAT was about 20 (80 scans). 
III. EXPERIMENTAL RESULTS AND DISCUSSION.

The resonance frequencles $\nu_{0}$ of the four investigated transitions in the absence of hyperfine structure are given in Table I, together with the measured frequencles of the hyperfine components. Recorder tracings with frequency scale for all transitions are shown in Fig. 4 .

The desired hyperfine coupling constants were derived from the fit of the experimental spectra to the theoretical ones obtained by diagonalizing the Hamiltonian matrix. The matrix elements of the hyperfine Hamiltonian have been calculated in the $\left|\left(\mathrm{J}_{Y \pm} I_{4}\right) F_{1} I_{F} M_{F}\right\rangle$ representation, which corresponds to the coupling scheme:

$$
\bar{J}_{\gamma \pm}+\bar{I}_{4}=\bar{F}_{1}, \bar{I}_{1}+\bar{I}_{2}+\bar{I}_{3}=\bar{I}, \bar{F}_{1}+\bar{I}=\bar{F},
$$

where $Y$ stands for the quantum numbers $K \tau$. Group theoretical considerations show that for the levels we are dealing with (A-levels), $I_{1}, I_{2}$, and $I_{3}$ are coupled to $I=3 / 2$ because of the Pauli exclusion principle. The matrix elements are given in the previous Chapter (Eqs. (35) and (42); the index \pm may be dropped if $k=0$ ). The relative intensities were calculated from the intensity matrix for low microwave field strength:

$$
\overline{\bar{I}}=\overline{\bar{R}}_{f}^{+} \overline{\bar{C}} \overline{\bar{R}}_{i}
$$

where $\overline{\bar{R}}_{1}$ and $\overline{\bar{R}}_{f}$ are the matrices diagonalizing the Hamiltonian matrix of the Initial and final state of the transition, respectively, and $\overline{\bar{C}}$ is the dipole moment matrix with elements:

$$
c_{q r}=\left\langle q\left|\mu_{E}\right| r\right\rangle
$$



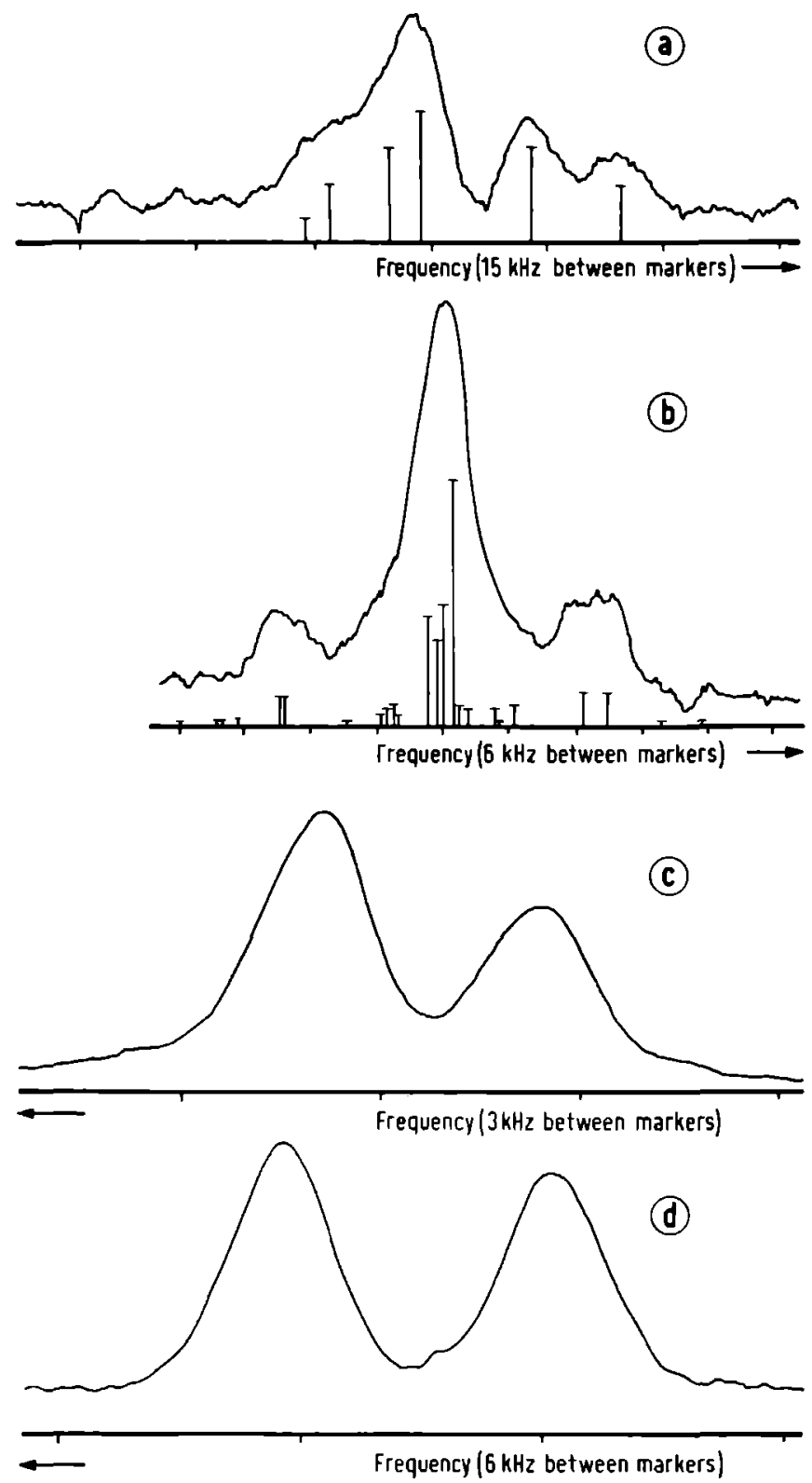

Fig.4. Recordings of the measured transitions: a: $(0,0,1) \rightarrow(1,0,1)$, $b:(2,1,3-) \rightarrow(2,1,3+)$, c: $(3,1,3-) \rightarrow(3,1,3+), \quad d:(6,1,3-) \rightarrow(6,1,3+)$. 
Table I. Measured (column 2) and unsplit line (column 3) frequencies of the $\mathrm{CH}_{3} \mathrm{OH}$ rotational transitions (1n $\mathrm{kHz}$ ).

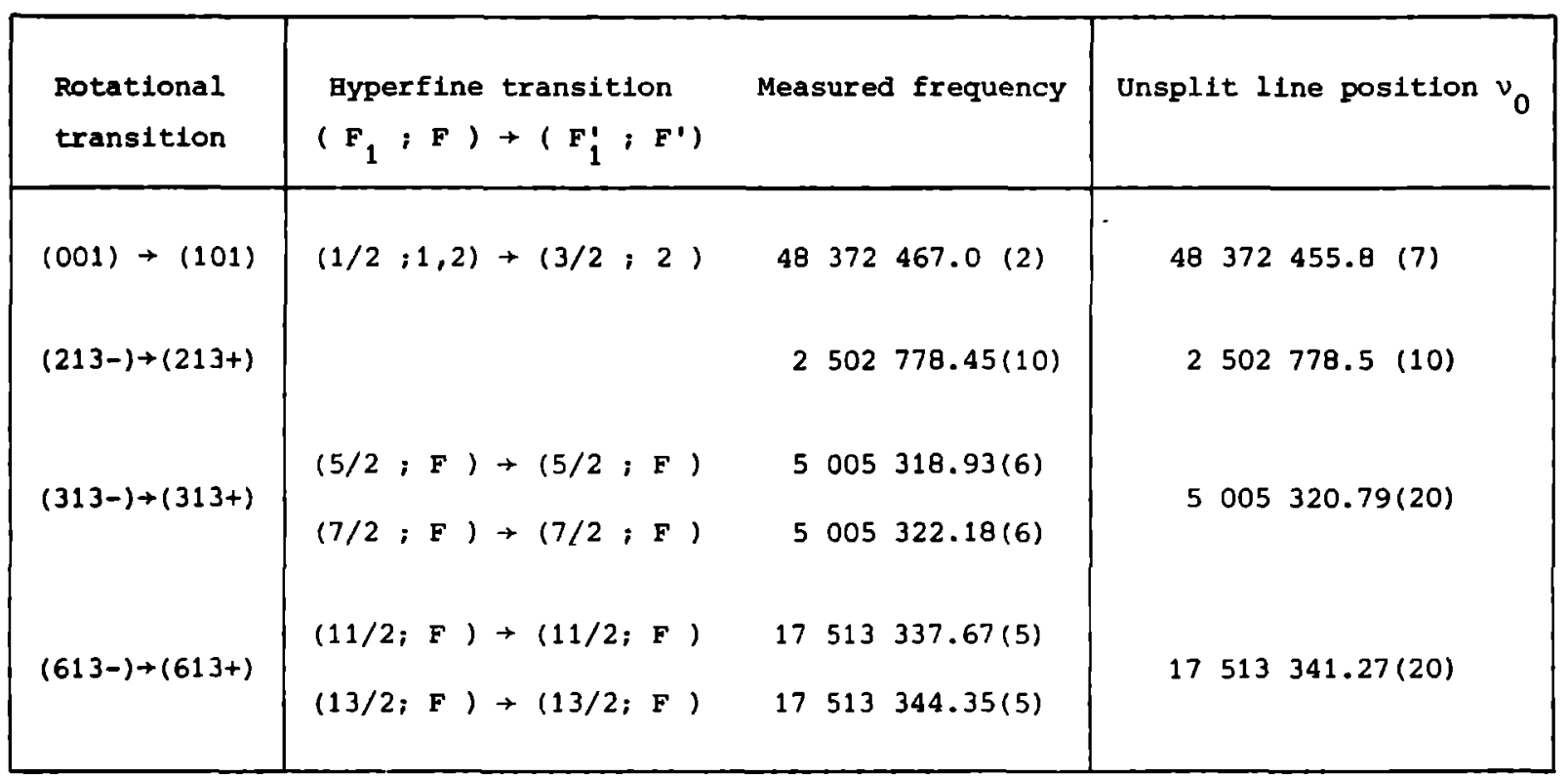


Herein $|q\rangle$ and $|r\rangle$ is the unperturbed final and initial state of the transition, respectively, and $\mu_{E}$ is the component of the electric dipole moment along the direction of the electric field of strength E. The matrix elements $C_{q r}$ are in the above representation, apart from insignificant constants, equal to (1) :

$$
\begin{aligned}
& C_{J F_{1} F_{i} J^{\prime} F_{1}^{\prime} F^{\prime}}=(-)^{J+F_{1}+F_{1}^{\prime}+F+I+I_{4}}\left[\left(2 F_{1}+1\right)\left(2 F_{1}^{\prime}+1\right)(2 F+1)\left(2 F^{\prime}+1\right)\right]^{\frac{1}{2}} \times \\
& \times\left\{\begin{array}{lll}
F_{1} & 1 & F^{\prime} \\
J^{\prime} & I_{4} & J^{1}
\end{array}\right)\left(\begin{array}{lll}
F^{\prime} & 1 & F^{\prime} \\
F_{1}^{\prime} & I & F_{1}
\end{array}\right\}
\end{aligned}
$$

If one of the two states Involved in the transition has $J$ equal to zero, the relative intensities of the hyperfine transitions are proportional to $2 \mathrm{~F}+1$ (5). If the hyperfine energy matrix is nearly diagonal in the above representation, the hyperfine transitions for a particular rotational transition can be denoted by $\left(F_{1} ; F\right) \rightarrow$ $\left(F_{1}^{\prime} ; F^{\prime}\right)$. All calculations were done on the IBM $360 / 50$ computer of the University.

The spectrum of the $(001) \rightarrow(101)$ transition consists of six hyperfine components, only partially resolved. The half-width is about $3 \mathrm{kHz}$. The coupling constants of the upper level (the lower level with $J=0$ has no hyperfine structure) are obtained by a curve fitting of the spectrum. The fitting parameters were all four coupling constants $\mathrm{C}_{\mathrm{J}_{\gamma}}^{(1)}, \mathrm{C}_{\mathrm{J}_{\gamma}}^{(2)}, \mathrm{D}_{\mathrm{J}_{\gamma}}^{(1)}$, and $\mathrm{D}_{\mathrm{J}_{\gamma}}^{(2)}$ and $\nu_{0}$. As starting values we took for the spin-spin coupling constants the calculated values, for the spin-rotation constants the value zero, and for $\nu_{0}$ a reasonable value, easily obtalned from the spectrum. The results are given in Table II, column 2. There is a rather good agreement 
Table II. Measured and calculated coupling constants (in $\mathrm{kHz}$ ). The tabulated value of $\Delta \mathrm{C}^{(2)}$ is the difference between the $C_{J \pm}^{(2)}$ constant of the $(+)$ and $(-)$ level of a $\Delta J=0, \Delta K=0$ transition.

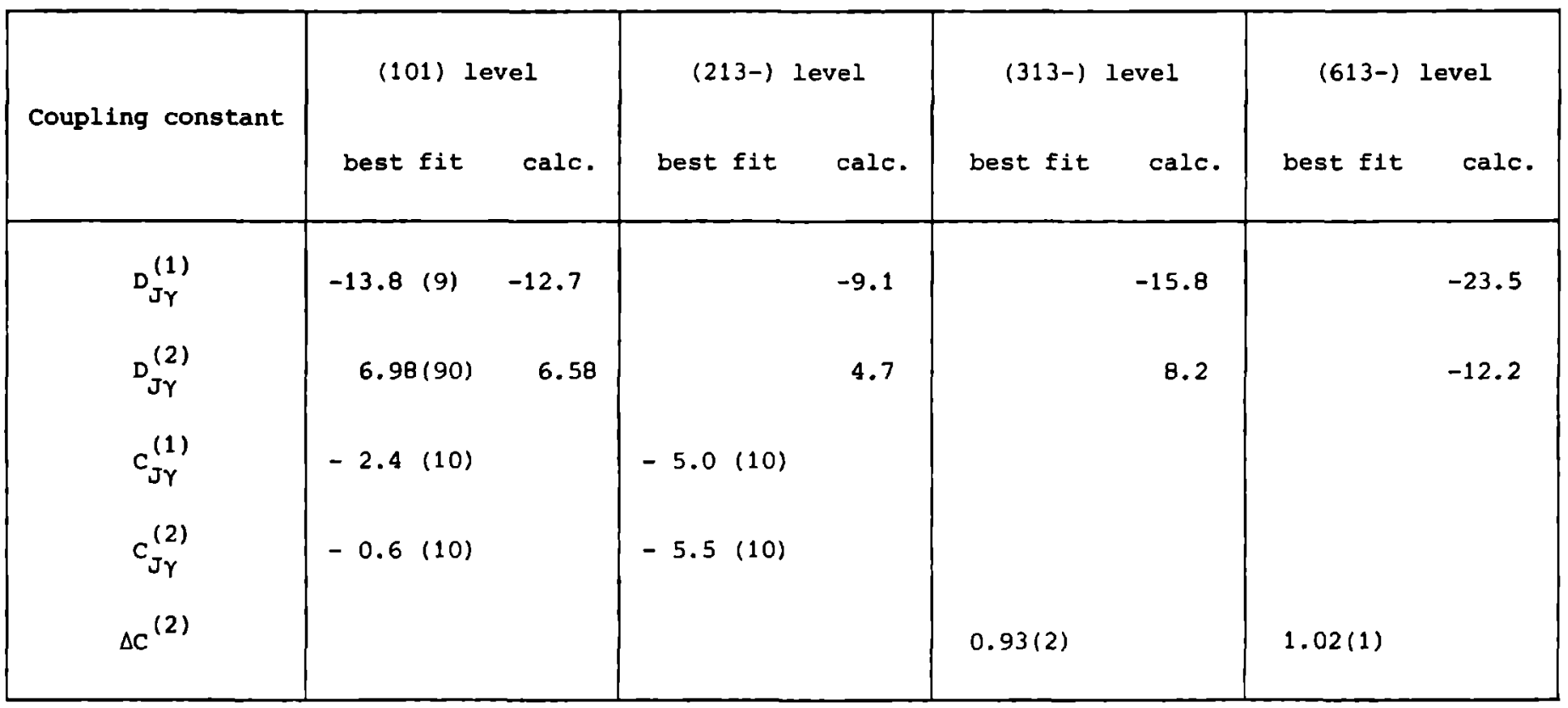


between the calculated and measured values of the spin-spin coupIIng constants. The calculated values depend on the internuclear distances which means that $D_{J Y}^{(2)}$ depends on the internal wave functions $\left|\tau_{K}\right\rangle$, but $D_{J Y}^{(1)}$ does not. The angle of internal rotation $\alpha$ is not involved in the mutual distances of the protons in the $\mathrm{CH}_{3}-$ group. The value of $D_{J Y}^{(2)}$ however increases only about 38 in the limit of entirely free internal rotation, and decreases in the same amount in the other limit of no internal rotation at all.

The spectra of the $J=3$ and 6 transitions have an almost identical structure, and are discussed together here. Besides the two strong lines, no other lines were observed in the frequency region of about $200 \mathrm{kHz}$, in spite of prolonged and careful searching. The calculated spectrum predicts elght strong $\Delta F=0, \Delta F_{1}=0$ transitions and a great number of much weaker transitions of other type: $\Delta F \neq 0$ and/or $\Delta \mathrm{F}_{1} \neq 0$. Consequently the two components in the measured spectra have to be ldentified with $\Delta F=0, \Delta F_{1}=0$ transitions. These lines are clearly not single lines. This is confirmed by the fact that the ratio of the line widths, obtalned with cavities of different lengths, is completely different from the ratio of the lengths of the cavities (6). The calculated value of the spin-spin coupling constants $\mathrm{D}_{\mathrm{J} Y \pm}^{(1)}$ is the same for the upper and for the lower level in our model; the calculated difference between the two values of $D_{J_{\gamma} \pm}^{(2)}$ is less than $0.5 \%$ and may be neglected. In order to fit the spectrum four parameters remaln to be varied: the spinrotation constants $\mathrm{C}_{\mathrm{J}_{Y \pm}}^{(1)}$ and $\mathrm{C}_{\mathrm{J}_{Y \pm}}^{(2)}$ of the upper and lower level. The hyperfine matrices of the upper and the lower level are the same except for the values $\mathrm{C}_{\mathrm{J} \pm}^{(1)}$ and $\mathrm{C}_{\mathrm{J} \pm^{-}}^{(2)}$. It is more convenient to ex- 
press the spin-rotation constants of the lower level as:

$$
\begin{aligned}
& c_{\mathrm{JKT}+}^{(1)}=c_{\mathrm{JKT}-}^{(1)}-\Delta C^{(1)} \\
& C_{\mathrm{JKT}+}^{(2)}=c_{\mathrm{JKT}-}^{(2)}-\Delta C^{(2)}
\end{aligned}
$$

From Eqs. (37), (49), and (50) of Chap.2 it is clear that $\Delta C^{(1)}$ and $\Delta C^{(2)}$ are independent of $J$ and equal to:

$$
\begin{aligned}
& \Delta C^{(1)}=\sum_{L=1}^{3}-\operatorname{Re}\left\langle 3_{1}\left|\left\{M_{L}\right\}{ }_{2}^{(2)}\right| 2_{-1}\right\rangle=-3 \operatorname{Re}\left\langle 3_{1}\left|\left\{M_{1}\right\}{ }_{2}^{(2)}\right| 2_{-1}\right\rangle, \\
& \Delta C^{(2)}=-\operatorname{Re}\left\langle 3_{1}\left|\left\{M_{4}\right\}{ }_{2}^{(2)}\right| 2_{-1}\right\rangle .
\end{aligned}
$$

We make now the following assumptions:

1) the hyperfine Hamlltonian is almost diagonal in the $\left|\left(J_{\gamma \pm} I_{4}\right) F_{1} I F M_{F}\right\rangle$ representation

11) $\Delta \mathrm{C}^{(1)}$ is small compared to $\Delta \mathrm{C}^{(2)}$.

With the assumptions stated above the calculated spectrum splits into two groups of lines with frequency $v_{1}=v_{0}-\frac{1}{2}(J+1) \Delta C^{(2)}$ and $v_{2}=v_{0}+\frac{1}{2} \mathrm{~J} \Delta \mathrm{C}^{(2)}$, respectively. The first group contains the four transitions with $F_{1}=J-\frac{1}{2}$, the second group the four transitions with $F_{1}=J+\frac{1}{2}$. The first assumption is fulfilled over a wide range of values of the spin-rotation coupling constants, as can be checked by explicit calculations. For values of the coupling constants outside this range one or more lines (but not all) of each group move to the center of the spectrum indicating a nonnegligible contribution of off-diagonal matrix elements. However, a good fit is not possible in this case. The second assumption can- 
not be proved in a straightforward way, but the measured spectrum could never be generated if $\Delta C^{(1)}$ is not relatively small. The frequency difference between both groups of lines 1s:

$$
\Delta v=v_{2}-v_{1}=\frac{1}{2}(2 J+1) \Delta C^{(2)}
$$

In order to determine also the sign of $\Delta c^{(2)}$ from the measured spectra it must be known which of the two investigated lines belongs to the $F_{1}=J-\frac{1}{2}$ and which to the $F_{1}=J+\frac{1}{2}$ transitions. From the relative intensities, tabulated by Townes (7) it follows immediately that the total intensity of the $F_{1}=J+\frac{1}{2}$ transitions is larger than that of the $F_{1}=J-\frac{1}{2}$ transitions. In the observed spectra ( $J=3$ and 6$)$ the line with the highest frequency has significantly higher intensity and hence can be identifled with the $F_{1}=J+\frac{1}{2}$ transitions. The observed $\Delta v$ values are:

$$
\begin{aligned}
& \Delta v=3.24(6) \mathrm{kHz} \text { for } \mathrm{J}=3 \text {, and } \\
& \Delta v=6.68(7) \mathrm{kHz} \text { for } \mathrm{J}=6 \text {. }
\end{aligned}
$$

The resulting $\Delta C^{(2)}$ values are given in Table II. These values do not agree within the quoted experimental error. This is not so surprising because the spectra of the two transitions cannot be put to colncidence by a simple change of the frequency scale; the observed lines are rather broad and not quite symetrical indicating that the involved transitions in each line are shifted in frequency. This may have its origin in the fact that one or both assumptions made above are not completely fulfllled. The two $\Delta c^{(2)}$ values agree within 108 and hence we write:

$$
\Delta C^{(2)}=-\operatorname{Re}<3_{1}\left|\left\{M_{4}\right\}_{2}^{(2)}\right| 2_{-1}>=0.98(9) \mathrm{kHz} .
$$


The observed spectra give no Information about the other coupling constants.

With the value of $\Delta C^{(2)}$ we calculated the values of $\nu_{0}$ for $J=3$ and 6. It is also given in Table I.

The spectrum of the $(213-) \rightarrow(213+)$ transitions consists of a strong main line and two satellite components originating fxom $\triangle F \neq 0$ and/or $\Delta F_{1} \neq 0$ transitions. To fit this spectrum we made use of the information obtalned from the $J=3$ and 6 transitions: $\Delta C^{(2)}=$ $0.98(9) \mathrm{kHz}$, and $\Delta \mathrm{C}^{(1)} \simeq 0$. Using this information and the calculated values of the spin-spin constants, only the spin-rotation coupling constants of the upper level have to be determined from the fit. The best-fit values are given in Table II. The absence of splitting of the main line into two components, as in the case of $J=3$ and 6 , is readily understood from the fact that for the fitted values of coupling constants, the hyperfine matrices are not diagonal.

IV. CONCLUSIONS.

The spin-rotation coupling constants of the (101) level are:

$$
\begin{aligned}
& \left.c_{101}^{(1)}=\frac{3}{2}<1_{0}\left|\left(M_{1}\right)_{x x}+\left(M_{1}\right)_{y y}\right| 1_{0}\right\rangle \\
& \left.C_{101}^{(2)}=\frac{1}{2}<1_{0}\left|\left(M_{4}\right)_{x x}+\left(M_{4}\right)_{y y}\right| 1_{0}\right\rangle .
\end{aligned}
$$

Consequently only the sum of $\left\langle 1_{0}\left|\left(M_{L}\right)_{X X}\right| 1_{0}\right\rangle$ and $\left\langle 1_{0}\left|\left(M_{L}\right)_{Y Y}\right| 1_{0}\right\rangle$ is determined. In fact this is the case for all transitions of $\mathrm{CH}_{3} \mathrm{OH}$. It would be interesting to determine the influence of $\tau_{K}$ upon $\left.{ }^{<\tau_{K}}\left|\left(M_{I}\right)_{X X}+\left(M_{L}\right)_{Y Y}\right| \tau_{K}\right\rangle$. However, it is very difficult to extract 
the spin-rotation coupling constant from $\Delta \mathrm{J}=0, \Delta \mathrm{K}=0$ transitions, because in this case the coupling constants of the upper and the lower level are almost equal. Thls causes the strongest hyperfine transitions to colnclde. Explicit calculations show that measurements on transitions wlth $\Delta J \neq 0$ or $\Delta K \neq 0$ would permit the determination of Individual coupling constants and their dependence on the internal wavefunction $\mid \tau_{K}>$.

REFERENCES.

(1) H. Bluyssen, Thesis 1968, Katholleke Universiteit, Nijmegen.

(2) J.A.Th. Verhoeven, Thesis 1969, Katholieke Universitelt, Nijmegen.

(3) C. Hulszoon, Thes1s 1966, Katholieke Untversiteit, Nijmegen.

(4) C. Huiszoon, Rev.Sci.Inst. 42,477 (1971).

(5) A.W. Ellenbroek, Quarterly Report No 30, Atomic and Molecular Research Group, Katholieke Universiteit, Nijmegen, (1971).

(6) Th. Lamers, Quarterly Report No 21, Atomic and Molecular Research Group, Katholieke Unlvers1te1t, Nijmegen, (1968).

(7) C. Townes and A.I. Schawlow, "Microwave Spectroscopy", McGraw Hill Book Company, Inc., New York (1955). 


\section{DISCUSSION}

\section{METHANOL.}

The magnetic hyperfine splitting of a rotational level of methanol can be described by four coupling constants: (1) the spin-spin coupling constant of the interactions between the magnetic moments in the frame and the three protons in the top, (2) the spin-spin coupling constants of the Interactions between the magnetic moments of the protons in the top alone, (3) the spin-rotation coupling constant of the Interaction of the magnetic moment of the proton in the frame with the magnetic fields produced by the internal and the overall rotation, and (4) the spin-rotation coupIing constant of the same interaction for the protons in the top. The second interaction vanishes for the levels of E-symmetry. As pointed out in Chap.2, all coupling constants can be described in terms of the components of the coupling tensors $\overline{\bar{D}}_{K}, \overline{\bar{M}}_{K}$, and $\bar{d}_{K}$ of the spin-spin, spin-overall rotation, and the spin-internal rotation interaction, respectively. Now we will discuss the possibility of determining the components of the tensors, rather than the coupling constants from investigated spectra. We restrict ourselves to the spin-rotation interaction, since the spin-spin coupling constants (and tensor components) can be calculated from the known molecular geometry and internal rotation wavefunctions - The agreement with the measured values for the (101) level, for example was quite good and also the same can be expected for the other ro- 
tational levels.

The spin-rotation coupling constants are given by Eqs. (37), (38), (49), and (50) of Chap.2 and can be rewritten as:

$$
\begin{aligned}
& \mathrm{C}_{\mathrm{JK} \tau( \pm)}^{(1)}=\sum_{\mathrm{L}}\left\{\frac{1}{2}\left[1-\frac{\mathrm{K}^{2}}{\mathrm{~J}(\mathrm{~J}+1)}\right]\left\langle\tau_{K}\left|\left(\mathrm{M}_{\mathrm{L}}\right)_{\mathrm{XX}}{ }^{+}\left(\mathrm{M}_{\mathrm{L}}\right)_{\mathrm{YY}}\right| \tau_{\mathrm{K}}\right\rangle \pm\right. \\
& \pm \frac{1}{2} \operatorname{Re}<3_{1}\left|\left\{M_{L}\right\}{ }_{2}^{(2)}\right| 2_{-1}>\delta(K, 1)+ \\
& \left.+\frac{K}{J(J+1)}\left[K<\tau_{K}\left|\left(M_{L}\right)_{z z}\right| \tau_{K}>-\frac{1}{2} R e<\tau_{K}\left|P\left(d_{L}\right)_{z}+\left(d_{L}\right) p\right| \tau_{K}>\right]\right\}
\end{aligned}
$$

where the sum runs over $L=1,2,3$ if $i=1$ and only over $L=4$ if $1=2$. The second term vanishes for E-levels. The contribution of the protons in the top $(L=1,2,3)$ is the same for each proton.

All tensor components are averaged over the internal wavefunctions, and hence only the coupling constants with the same $\tau$ and $K$ can be used for the determination of the tensor components. It would be interesting to determine the dependence of the tensor components on the state $\left|\tau_{K}\right\rangle$ of internal rotation. It can be shown by an explicit calculation that the spin-spin coupling constant $D_{J K \tau \pm}^{(2)}$ is nearly independent on the internal wavefunctions, but this does not 1mply that the same holds for the components of $\overline{\bar{M}}_{L}$ and probably also for the term ( $\left.p d_{z}+d_{z} p\right)$, since it is caused completely by the internal rotation. The contribution, for example, of $\left\{\mathrm{D}_{\mathrm{KL}}\right\}_{2}^{(2)}$ to the spin-spin coupling constant: $\operatorname{Re}<3_{1}\left|\left[D_{K L}\right\}_{2}^{(2)}\right| 2_{-1}>$ for $A-1$ evels with $K=1$, is very small because of the small dependence on the angle $\alpha$, while the analogous contribution of $\overline{\bar{M}}_{4}$ to the spin-rotation coupling constant (Re<3 $\left|\left\{M_{4}\right\}_{2}^{(2)}\right| 2_{-1}>$ ) is about $1 \mathrm{kHz}$ (Chap. 3 ) indicating a much larger dependence of the components of $\overline{\bar{M}}_{4}$ on $\alpha$. 
If sufficient transitions are investigated and the related coupling constants determined, the following quantities might be obtained under optimum conditions:

1) $<\tau_{K}\left|\left(M_{L}\right)_{X x}+\left(M_{L}\right)_{Y Y}\right| \tau_{K}>$,

11) $\operatorname{Re}<3_{1}\left|\left(M_{L}\right\}{ }_{2}^{(2)}\right| 2_{-1}>$, and

111) $\left.K<\tau_{K}\left|\left(M_{L}\right)_{z Z}\right| \tau_{K}>-\frac{1}{2} R e<\tau_{K}\left|p\left(d_{L}\right)_{z}+\left(d_{L}\right)_{z} p\right| \tau_{K}\right\rangle$

The components $\left(M_{L}\right)_{X x}$ and $\left(M_{L}\right)_{Y y}$ can never be determined separately, but only as a sum. This is an inherent property of the symmetric top molecules, and $\mathrm{CH}_{3} \mathrm{OH}$ is treated as such, which is a very good approximation.

The second term $\operatorname{Re}<3_{1}\left|\left\{M_{L}\right\}_{2}^{(2)}\right| 2_{-1}>$ exists only for A-type levels with $K=1$, and can be determined only by measuring the spectra of A-type rotational transitions in which one or both involved states is a level with $\mathrm{K}=1$. The term is generated only by the internal rotation and has been already determined (Chap.3).

The most interesting term is, of course, the quantity $\left.\operatorname{Re}<\tau_{K}\left|p\left(d_{L}\right)_{z}+\left(d_{L}\right)_{z} p\right| \tau_{K}\right\rangle$ which describes the spin-internal rotation interaction. Unfortunately this term cannot be determined Independently, but only in combination with the term $-2 K<\tau_{K}\left|\left(M_{L}\right)_{Z Z}\right| \tau_{K}>$. If and only if the expectation values of $\left(p d_{z}+d_{z} p\right.$ ) and $M_{z z}$ are 1ndependent on $K$ (which is open for experimental verification) the two contributions can be separated by measuring the coupling constants of levels with different K-values.

It is clear that many transitions have to be investigated to get more insight in the varlous coupling tensors and their depen- 
dence on the internal rotation. A large number of transitions in the centimeter and millimeter wave region are suitable for this purpose. Recently some of these transitions have been investigated, the E-type transitions $(2,1,2) \rightarrow(2,2,1)$ and $(5,1,2) \rightarrow(5,2,1)$ at about $25 \mathrm{GHz}$ by the author (1) and the $(2,0,2) \rightarrow(3,1,1)$ at about $12 \mathrm{GHz}$ by Gaines (2) with a beam-maser spectrometer and the A-type transition $(1,0,1) \rightarrow(2,0,1)$ at about $97 \mathrm{GHz}$ by Bicanic (3) with a beam-absorption spectrometer. Unfortunately the resolving power of these spectrometers was insufficient to resolve the hyperfine structure completely (some lines were partially split, others only broadened by the hyperfine interactions). A continued program on the hyperfine structure of methanol will require spectrometers with a resolving power increased by a factor of 5-10. A beam-maser spectrometer with two cavities for the centimeter region or two Fabry-Perot type interferometers for the millimeter region might be very useful (Ramsey patterns).

Another possibility is to investigate partially deuterated species of the methanol molecule $\left(\mathrm{CH}_{3} \mathrm{OD}, \mathrm{CD}_{3} \mathrm{OH}\right)$. The introduced electric quadrupole interaction of the deuterium nucleus is in general an order of magnitude larger than the other hyperfine interactions. It causes a large splitting of the hyperfine spectrum into several I ines, which in turn are split by the other hyperfine interactions. Because of the small nuclear g-factor of the Dcompared to the H-nucleus (a factor of six), it will be hard to measure the spin-rotation interaction of the deuterium nucleus. Apart from the electric quadrupole interaction, $\mathrm{CH}_{3} \mathrm{OD}$ is most suitable to measure the spin-rotation interaction of the protons in the 
top, and $\mathrm{CD}_{3} \mathrm{OH}$ to measure the spin-rotation interaction of the proton in the frame.

At this moment, no ab-initio calculation of the electronic structure of methanol is avallable. A continued program on the hyperfine structure might stimulate such calculation.

II. OTHER MOLECULES.

Hydrogen peroxide $\left(\mathrm{H}_{2} \mathrm{O}_{2}\right)$ is a favourable molecule for the investigation of hyperfine structure, since it is the most simple internal rotor. The hyperfine splitting of a rotational level can be described in terms of only two coupling constants, namely the spinspin coupling constant of the two protons and the spin-rotation coupling constant of one proton, which is equal to that of the other. Because of its relative simplicity the molecule is more accessible for ab-initio calculation of the electronic structure (4). However, there are only a few identifled transitions in the ground vibrational state known in the easely accessible microwave region.

Both $\mathrm{CH}_{3} \mathrm{OH}$ and $\mathrm{H}_{2} \mathrm{O}_{2}$ are internal rotors with relatively high potential barriers (about $380 \mathrm{~cm}^{-1}$ ). This implies that the internal motion is more a libration rather than a rotation. It is expected, however, that the influence on the hyperfine interactions, especially the spin-internal rotation interaction, is more pronounced if the internal rotation is nearly free. More information about the internal rotation and hyperfine interactions could be obtained if the investigations are extended to internal rotor molecules with relatively low potential barriers. However, it will be hard to find suitable molecules, since apart from $\mathrm{CH}_{3} \mathrm{OH}$ and $\mathrm{H}_{2} \mathrm{O}_{2}$ most internal 
rotor molecules are rather large and intricate. It is expected, that in general the spectra of these molecules will contain clusters of hyperfine transitions because of the presence of many nuclear spins, Resolution of these clusters will require spectrometers of very high resolving power. Moreover, the intensity of the rotational transitions will be rather weak, because of the very large number of rotational levels between which transitions may take place. For a given rotational transition this intensity will be distributed between many allowed hyperfine transitions, leading to very serlous sensitivity problems. The prospects of investigating the structure of internal rotor molecules other than methanol and hydrogen peroxide look not very promising.

REFERENCES.

(1) J.E.M. Heuvel, Quarterly Report No 37, Atomic and Molecular Research Group, Katholleke Universitelt, Nifmegen, (1972).

(2) L. Gaines, Columbla University, New York, private communtcation.

(3) D.D. Bicanic, Quarterly Report No 36, Atomic and Molecular Research Group, Katholleke Universitelt, N1Jmegen, (1972).

(4) R.B. Davidson and L.C. Allen, J,Chem.Phys. 55, 519 (1970). 
In dit proefschrift worden de magnetische hyperfijne interakties in interne rotor molekulen en de metingen verricht aan het methanol molekuul $\left(\mathrm{CH}_{3} \mathrm{OH}\right)$ beschreven.

B1f het begin van het huldige onderzoek was er geen theorie beschikbaar over de hyperfijne struktuur in interne rotor molekulen. In Hoofdstuk 2 wordt de Hamlltonlaan met 1nbegrip van hyperfijne Interakties afgeleld. De Hamlltoniaan geldt slechts voor molekulen met één interne vrijheidsgraad zoals methanol, maar kan gemakkelijk uitgebreid worden voor interne rotor molekulen met meerdere interne vrifheldsgraden. De magnetische hyperfijne interakties bestaan uit de spin-spin interakties en de spin-rotatie interakties. De spin-spin Interaktie is de wisselwerking tussen de magnetische dipool momenten van de kernen onderling. Deze lnteraktle hangt af van de onderlinge afstanden tussen de kernen en wordt derhalve beinvloed door de interne rotatie. De spin-rotatle interaktie is het gevolg van de wisselwerking tussen de magnetische dipool momenten van de kernen en het effektieve magnet1sche veld ter plaatse van de kernen. Dit magnetische veld wordt veroorzaakt, zowel door de rotatie van het molekuul als geheel ("overall" rotatie), als door de interne rotatie. Er $z 1 j n$ dus twee bljdragen tot de spin-rotatie interaktie, ramelijk de spin-interne en de spln-"overall" rotatle interaktie. Met behulp van sferische operatortechnieken werden de hyperfijne opsplitsingen van de rotatie niveaus van methanol berekend in termen van hyperfijne koppelingskonstanten. Molekulaire groepentheorie bleek een belangrifk hulpmiddel hierbif. Expliciete uitarukkingen 
konden worden afgeleid voor de koppelingskonstanten van methanol.

De hyperfijne struktuur op de $(J, 1,3-) \rightarrow(J, 1,3+)$ rotatie overgangen van methanol voor $J=2,3$ en 6 werd onderzocht met een bearn-maser spektrometer en op de $(0,0,1) \rightarrow(1,0,1)$ overgang met een beam-absorptie spektrometer (Hoofdstuk 3). De beam-maser spektrometer werd gebouwd door Bluyssen (1968) en de beam-absorptie spektrometer door Huiszoon (1966). In belde spektrometers wordt een hoog oplossend vermogen verkregen door gebruik te maken van molekulaire bundels, waardoor de Doppler verbreding van de spektraallijnen sterk wordt gereduceerd. Ter vergroting van de gevoeligheid werden belde spektrometers uitgebreid met een systeem om "time-averaging" technieken te kunnen toepassen. De gemeten spektra werden geinterpreteerd met behulp van de in Hoofdstuk 2 afgeleide theorle en de relevante koppelingskonstanten konden worden bepaald. De spin-rotatle koppelingskonstanten kunnen worden u1tgedrukt In de komponenten van Cartesische tensoren. In Hoofdstuk i worden de mogelijkheden onderzocht om deze tensorkomponenten voor het methanol molekuul te bepalen. Bovendien worden de vooruitzichten besproken om de hyperfijne struktuur te onderzoeken van andere interne rotor molekulen dan methanol. 
De door Kukolich gemeten hyperfijne spektra van ${ }^{15} \mathrm{NH}_{3}$ zijn geinterpreteerd met foutieve uitdrukkingen voor de matrixelementen van de spin-spin interaktie tussen de waterstofkernen. Het verdient aanbeveling deze spektra opnieuw te analyseren.

S.E. Kukolich, Phys. Rev., 172, 59 (1968).

\section{II}

De bewering van Mariot, dat de nulmatrix een triviale representatie van een groep vormt, is onjuist.

L. Mariot, 'Groupes finis de symmetrie et recherche de solutions de l'équation de Schrödinger', Dunod, Paris, 1959 (p. 10).

\section{III}

De mogelijkheid, dat kleine clusters van waterstofmolekulen metallieke eigenschappen hebben, is aan emstige twijfel onderhevig.

\section{IV}

De deoor Rogers en Barrett uitgevoerde berekening van de waarschijnlijkheid, dat interstellaire $\mathrm{OH}$ radikalen binnen het ${ }^{2} \Pi_{3 / 2}, J=3 / 2 \Lambda$-doublet een door elektronen of ionen geinduceerde overgang maken, is ten dele onjuist.

A.E.E. Rogers en A.H. Barrett, Ap. J., 151, 163 (1968). 
Het verband tussen de door Kelsey gemeten sterkten van de 'prebreakdown' stroom en de Rontgenemıssie blijkt verschillend te zijn voor verschullende vacuumsystemen. De door de auteur gegeven verklaring met behulp van het Townsend mechanisme is overbodig en onwaarschunnlık op grond van de afwijkende experimentele omstandigheden.

T. Kelsey, J. Phys. D Appl. Phys., 5, 569 (1972).

De door Cross et al. gegeven waarden voor de totale botsingsdoorsneden bi] zuivere multıpoolinteraktıes zıjn zeer onnauwkeurig ( $50 \%)$.

R.J. Cross, E.A. Gislason, en D.R. Herschbach, J. Chem. Phys., 45, 3582 (1966).

VII

Autorijscholen dienen uit didaktısch oogpunt meer dan tot nu toe gebruik te maken van veilıgheidsgordels.

\section{VIII}

In het voortgezet onderwijs, zeker voor het vak natuurkunde, is geen plaats voor drie verschillende leraarsgraden. 

